\title{
Critical tests of stellar evolution in open clusters. I. New photometry and radial velocities for NGC $3680^{\star}$
}

\author{
B. Nordström, J. Andersen and M.I. Andersen \\ Niels Bohr Institute for Astronomy, Physics, and Geophysics, Astronomical Observatory, Juliane Maries Vej 30, DK- \\ 2100 Copenhagen, Denmark \\ Received November 10, 1995; accepted January 5, 1996
}

\begin{abstract}
We present new CCD photometry in the $b$ and $y$ colours of the Strömgren uvby system for 310 stars in a $13^{\prime} \times 13^{\prime}$ field centered on the intermediate-age open cluster NGC 3680 . Careful cross-checks indicate that previously published $B V$ photometry of NGC 3680 is affected by random and/or systematic errors precluding its use in critical comparison with theoretical isochrone computations. Detailed notes on several individual stars are given. In addition, we present $\approx 400$ new photoelectric radial-velocity observations of 109 stars obtained with the CORAVEL scanner during the period 1988-1994. These data allow substantially complete identification of member and non-member stars in the field, and of spectroscopic binaries in both groups. Rotational velocities have also been derived for the programme stars, and our velocity variability criteria for stars of all rotations are described. The further astrophysical discussion of the data, including the definition of radial-velocity membership criteria, theoretical isochrone fitting, and the dynamical state of the cluster and the origin of its "bimodal turnoff", will appear in a separate paper (Nordström et al. 1996).
\end{abstract}

Key words: stars: evolution — stars: fundamental parameters — stars: HR diagram — stars: kinematics — open clusters and associations: NGC 3680

\section{Introduction}

Intermediate-age and old open star clusters are classic test objects in a variety of stellar and Galactic research fields. As ensembles of stars of identical age and chemical composition, star clusters are favourite test objects for stellar evolution models (e.g. VandenBerg 1983, 1985; Demarque et al. 1994). Clusters are also key objects in probing the structure and evolution of the galactic disk (e.g. Janes \& Phelps 1994). With the increasing awareness of the complexities of the actual evolutionary history of the Galactic disk and its stars, demands on the quality and quantity of observational data are continually increasing.

Most investigations using cluster colour-magnitude diagrams (CMDs) rest on the basic, if sometimes implicit assumption that an accurate sequence of single cluster stars can be defined in a well-observed and well-calibrated CMD. Real CMDs, however, inevitably contain an admixture of field and binary stars which complicate the inter-

Send offprint requests to: B. Nordström:

email: birgitta@astro.ku.dk

${ }^{\star}$ Based on observations obtained with the Danish 1.5-m telescope at the European Southern Observatory, La Silla, Chile

${ }^{\star \star}$ Tables 1 and 2 are also, and Table 3 only, available in electronic form at the CDS via anonymous ftp to 130.79.128.5. pretation; and photometry, whether photographic, photoelectric, or CCD, is not always perfect. Sparsely populated open clusters present special difficulties if the contrast from the field star background is low and binary stars are frequent.

Proper motion studies of sufficient accuracy are important to separate cluster and field stars, but still exist for only a handful of nearby clusters and leave close binaries undetected. In contrast, radial-velocity studies give information on both cluster membership and duplicity and do not require first-epoch plates. Radial-velocity data have already proved to be of considerable value in, e.g. M 67 (Mathieu \& Latham 1986; Mathieu et al. 1986; Nissen et al. 1987) and NGC 752 (Daniel et al. 1994). This paper reports the first results of a program to provide such precise radial-velocity data and, where needed, new photometry for selected southern open clusters.

NGC 3680, the first object to be discussed in this program, is an intermediate-age (approx. 2 Gyr) cluster of metallicity slightly above solar (Eggen 1969; Nissen 1988 $=\mathrm{N} 88$ ). CCD uvby photometry of the central parts of NGC 3680 was published by Anthony-Twarog et al. (1989; ATTS), photoelectric $u v b y-\mathrm{H}_{\beta}$ photometry for 33 stars by N88, and CCD and photographic $B V$ photometry in a large field centered on NGC 3680 by Anthony-Twarog et 
al. (1991; AHTC). Very recently, a proper-motion study of the cluster was also published by Kozhurina-Platais et al. (1995).

Our comparisons between the photometric data from the above sources revealed a number of systematic and individual inconsistencies precluding their use for accurate comparison with theoretically computed isochrones. Therefore, we have obtained new CCD photometry in the Strömgren uvby system in a $13^{\prime} \times 13^{\prime}$ field centered on NGC 3680. These results are reported and compared to previous photometry in the present paper (Sect. 2).

No radial-velocity study of sufficient accuracy to segregate cluster and field stars and detect the majority of spectroscopic binaries is available for NGC 3680, except for the recent survey of 15 red-giant candidates by Mermilliod et al. (1995). Accordingly, the bulk of our observational effort has been devoted to a radial-velocity survey of the cluster and the field in which it is imbedded. These data are also presented in the present paper (Sect. 3). With the new photometry, radial velocities, and proper motions, a comprehensive discussion of the cluster becomes possible, which is the subject of Paper II in this series (Nordström et al. 1996). Preliminary results from our radial-velocity survey were made available by us for the discussions in ATTS and AHTC (see also Nordström \& Andersen 1991 and Andersen \& Nordström 1991).

At present, no less than four different numbering systems exist for the stars in NGC 3680: That by Eggen (1969), identified here by the prefix E, the two different systems introduced by AHTC and ATTS (who give cross-references), and the numbering used in the propermotion study by Kozhurina-Platais et al. (1995), who also give cross-references to Eggen and AHTC. For the brighter stars, Eggen numbers have been used most frequently in previous papers on NGC 3680; as the AHTC list was the most exhaustive available when the bulk of our observations were made, we have primarily used that to identify fainter stars and stars outside the field studied by Eggen.

Three close doubles were omitted by AHTC due to crowding, but resolved on the CCD frames of ATTS and ourselves; we have included them in the AHTC system by adding 4000 to their ATTS numbers (Nos. 6027, 6028, and 7020). A few close companions to stars with Eggen numbers have been discovered here and labelled with suffixes: 21B (a companion to $21 \mathrm{~A}$ ), 30A, and 59A. For the stars in our CCD frames for which no previous Eggen or AHTC numbers existed, we have assigned running numbers in order of increasing right ascension, adding 9000 to avoid overlap with the previous AHTC system.

Thus, a unified numbering system will be used in the remainder of this paper (see Table 1) and in Paper II: Numbers below 100 refer to Eggen (1969), between 1000 and 5000 to AHTC, between 6000 and 8000 to ATTS (subtract 4000 to get the ATTS numbers as published), and numbers above 9000 to the stars newly observed in our CCD photometry. For convenience, Table 2 gives cross-references between this system and the numbers of Kozhurina-Platais et al. (1995) for stars measured in our radial-velocity survey.

Finally, with contemporary astronomical technology, accurate coordinates are more useful and less ambiguous stellar identifications than labelled finding charts. Accordingly, we have determined equatorial coordinates for all stars measured on our CCD frames, using all available stars from the HST Guide Star Catalog (Lasker et al 1990) as reference stars. The resulting accuracy is about 0 .'4, and comparison with stars in Kozhurina-Platais et al. (1995) shows agreement to better than $0 . \prime 5$ in both coordinates. Stars in AHTC, but outside the CCD field, have been identified in the HST Guide Star Catalog, and coordinates for these stars are given at the end of Table 1.

\section{Photometric data for NGC 3680}

In preparation for the discussion of the CMD of NGC 3680 , we performed a number of consistency checks on the published photometry for NGC 3680 . The results of these checks (cf. Sect. 2.2 below) indicated that none of the existing photometry was both extensive and reliable enough to be useful in isochrone fitting at the intended level of precision. Therefore, new CCD photometry was obtained as described in the following.

\subsection{New CCD photometry}

New CCD photometry was obtained with the $1.54 \mathrm{~m}$ Danish telescope on La Silla, on the night Jan. 25/26, 1995, using the wide field instrument Danish Faint Object Spectrograph \& Camera (DFOSC; see Andersen et al. 1995a). DFOSC, which is a combined focal reducer and spectrograph, is equipped with a thinned $2048 \times 2048,15-\mu$ pixel Loral CCD detector. With a QE above $70 \%$ in the whole spectral range 3000-8000 $\AA$, the response of the detector permits a good match to the standard filter-defined passbands. The final pixel scale is $0{ }^{\prime \prime} 391$ per pixel, giving a field of view of 13 ' 35 . Thus, nearly the entire cluster itself and a good area of the surrounding field are covered by a single exposure.

On the night of observation, the weather was photometric and the seeing around 1."5. In each of the $b$ and $y$ filters, ten 30-s. exposures and four 90-s. exposures were obtained. Between exposures, the telescope was offset by $3^{\prime \prime}$ to $3^{\prime}$. Half of the exposures were obtained in one rotator position, the other half in the opposite rotator position. In each filter, six sky flats were obtained, covering both rotator positions.

All images were bias subtracted and flat field corrected by the combined sky flats, using IRAF ${ }^{1}$. In this generally 
Table 1. Coordinates and CCD by photometry in the Strömgren system for 310 stars in a $13^{\prime} \times 13^{\prime}$ field centered on NGC 3680. For an additional 42 stars observed with CORAVEL, but outside the CCD field, coordinates from the HST GSC are listed at the end of the table

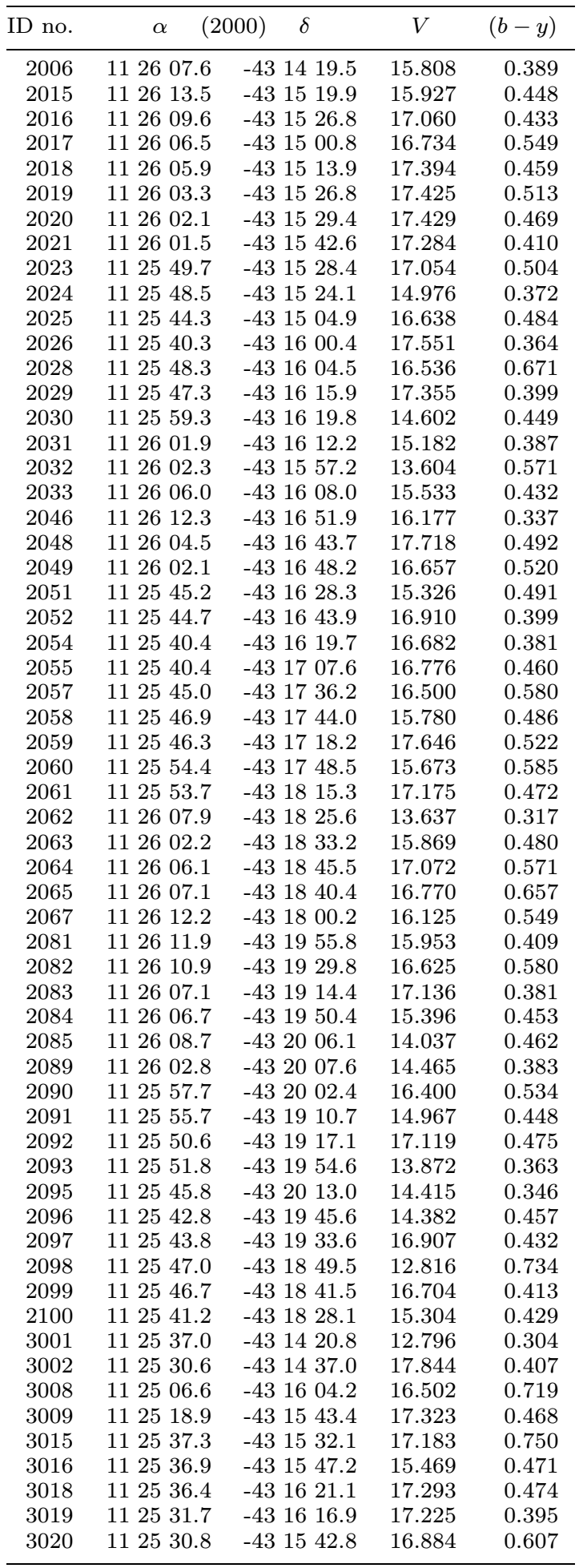

Table 1. continued

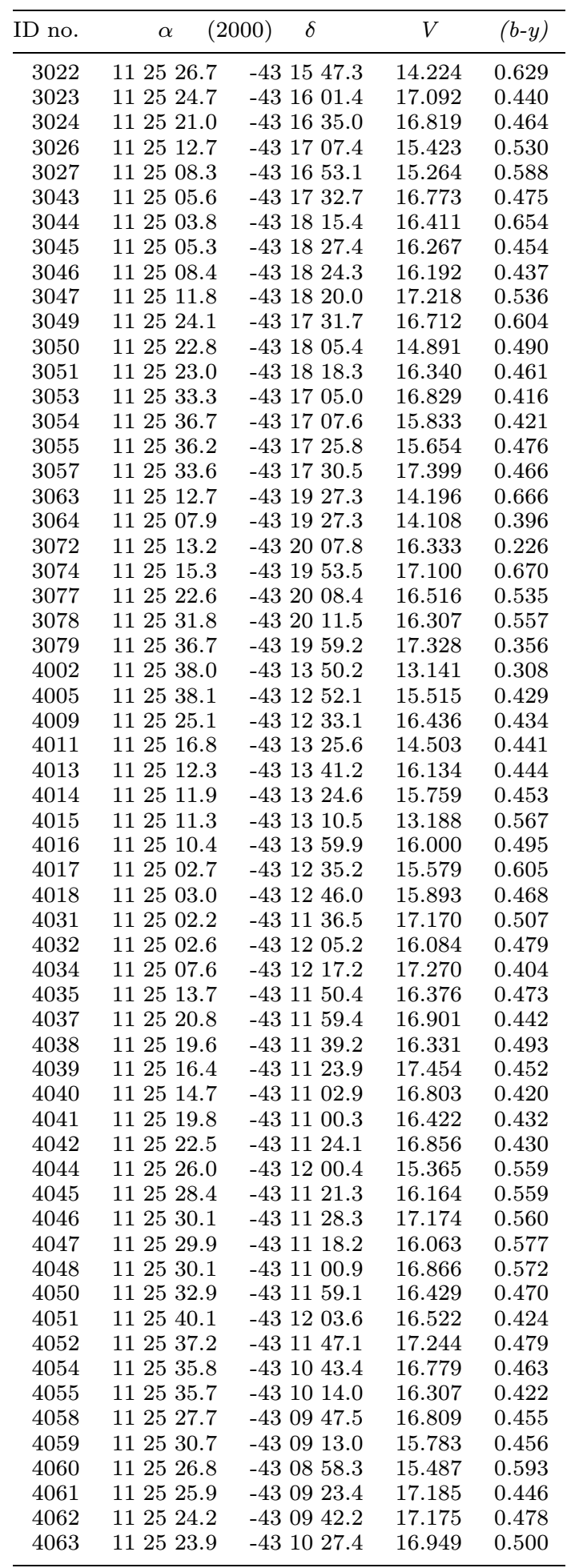


Table 1. continued

\begin{tabular}{|c|c|c|c|c|}
\hline ID no. & $\alpha$ & $0)$ & $V$ & $(b-y)$ \\
\hline 4065 & 112519.5 & -431010 & & 40 \\
\hline 4066 & 112521.4 & -4309 & & 473 \\
\hline 4067 & 112522.2 & $\begin{array}{lll}-43 & 09 & 19.1\end{array}$ & 16.436 & 650 \\
\hline 4069 & 12520.0 & -430910.5 & & \\
\hline 4071 & 12516.5 & -431013.8 & 87 & 602 \\
\hline 4073 & 112510.3 & -431043.0 & 16.301 & 559 \\
\hline 4074 & 112506.7 & -431032.6 & 16.151 & .491 \\
\hline 4075 & 112506.7 & -431026.0 & 17.096 & 647 \\
\hline 4075 & 112506.3 & -4311 & & 416 \\
\hline 4077 & $\begin{array}{lll}11 & 25 & 03.7\end{array}$ & -4310 & & 430 \\
\hline 4088 & 112502.6 & -430756.8 & 13.846 & .505 \\
\hline 4089 & 112506.7 & -430844.5 & & .445 \\
\hline 4090 & 112506.1 & -4309 & & 475 \\
\hline 4091 & 112509.2 & -43092 & & 466 \\
\hline 4093 & 112510.5 & -43074 & & 377 \\
\hline 4094 & 112515.1 & $\begin{array}{lll}-43 & 07 & 44.4\end{array}$ & 58 & .579 \\
\hline 4095 & 112517.6 & -43083 & & 463 \\
\hline 4096 & 12525.8 & -4308 & & 775 \\
\hline 4097 & 12527.7 & $\begin{array}{lll}-43 & 08 & 27.2\end{array}$ & 27 & 538 \\
\hline 4098 & 112534.0 & -4308 & 78 & .450 \\
\hline 4099 & 112533.6 & -43080 & & .720 \\
\hline 4100 & 12540.0 & -4308 & & 61 \\
\hline 4101 & 12540.2 & -4308 & & 41 \\
\hline 4102 & 12534.6 & -43071 & 92 & \\
\hline 4103 & 12529.8 & -43065 & & 409 \\
\hline 4104 & 112522.2 & -43071 & & \\
\hline 4. & 12520.5 & -43072 & & 18 \\
\hline 4116 & 12506.2 & -43071 & 59 & 115 \\
\hline 6016 & L 2537.4 & -4314 & & 501 \\
\hline 6027 & 112542.8 & -43 & & .353 \\
\hline & 25 & -4313 & & \\
\hline 7020 & 12532.6 & -43155 & & 85 \\
\hline 7021 & 2532.8 & -4315 & & .519 \\
\hline 9001 & 112613.8 & -43 & & 35 \\
\hline & L 2613.2 & -4313 & & \\
\hline 9 & L 2612.7 & -4311 & & \\
\hline 9004 & 12611.3 & -4311 & & 48 \\
\hline 9 & 2609.6 & -43133 & & 353 \\
\hline 9006 & 112609.2 & -4 & & 36 \\
\hline & .2 & -431 & & \\
\hline 9 & 2606.2 & -43181 & 51 & 80 \\
\hline 9009 & 12602.1 & -43121 & & 335 \\
\hline 9010 & 112557.8 & -43194 & & .767 \\
\hline & 12556.3 & -4313 & & 537 \\
\hline & .3 & 12 & & \\
\hline & 25 & -43075 & 60 & 08 \\
\hline 9 & 12553.0 & -43143 & & 134 \\
\hline & 112550.6 & -43090 & & 642 \\
\hline & 7 & & & \\
\hline & 1254 & -43092 & 73 & 11 \\
\hline & 12548.5 & -4308 & & 28 \\
\hline 9 & 112547.5 & -43170 & 95 & .461 \\
\hline & 112543.0 & -43185 & 70 & 0.442 \\
\hline & 12539.2 & $\begin{array}{lll}-43 & 161\end{array}$ & & 77 \\
\hline & 2533.8 & -43190 & & \\
\hline & 112524.2 & -43192 & 48 & 532 \\
\hline 9024 & 112523.8 & -431059.5 & 17.243 & 0.929 \\
\hline & 112522.8 & $\begin{array}{lll}-43 & 160\end{array}$ & 17.665 & 0.656 \\
\hline & 12520.9 & -43132 & & 0.617 \\
\hline 9027 & 112519.8 & -431154.7 & 16.748 & 0.697 \\
\hline
\end{tabular}

Table 1. continued

\begin{tabular}{|c|c|c|c|c|c|}
\hline ID no. & $(2$ & 00) & $\delta$ & V & $(b-y)$ \\
\hline 9028 & 112519.1 & -43 & 1546.7 & 17.360 & 0.499 \\
\hline 9029 & 112518.6 & -43 & 1717.1 & 17.020 & 0.733 \\
\hline 9030 & 112518.0 & -43 & 1446.7 & 17.681 & 0.583 \\
\hline 9031 & 112517.4 & -43 & 1112.2 & 18.185 & 0.538 \\
\hline 9032 & 112516.0 & -43 & 1804.5 & 17.626 & 0.531 \\
\hline 9033 & 112515.2 & -43 & 1637.3 & 17.999 & 0.487 \\
\hline 9034 & 112513.3 & -43 & 1418.0 & 17.848 & 0.604 \\
\hline 9035 & 112513.1 & -43 & 1704.0 & 17.465 & 0.483 \\
\hline 9036 & 112511.7 & -43 & 1840.0 & 17.740 & 0.656 \\
\hline 9037 & 112508.3 & -43 & 1935.2 & 17.488 & 0.723 \\
\hline 9038 & 112507.9 & -43 & 1146.7 & 17.665 & 0.600 \\
\hline 9039 & 112507.3 & -43 & 1836.2 & 17.708 & 0.656 \\
\hline 9040 & 112506.7 & -43 & 1258.6 & 17.572 & 0.513 \\
\hline 9041 & 112502.4 & -43 & 0911.9 & 17.218 & 0.774 \\
\hline 1 & 112501.5 & -43 & 1023.4 & & \\
\hline 3 & 112500.0 & -43 & 1524.0 & & \\
\hline 50 & 112624.8 & -43 & 1525.8 & & \\
\hline 53 & 112636.4 & -43 & 1514.5 & & \\
\hline 54 & 112639.7 & -43 & 1443.3 & & \\
\hline 55 & 112641.5 & -43 & 1018.8 & & \\
\hline 60 & 112620.7 & -43 & 1308.0 & & \\
\hline 61 & 112621.2 & -43 & 1330.8 & & \\
\hline $62(\mathrm{~A})$ & 112633.4 & -43 & 2232.6 & & \\
\hline $63(\mathrm{~B})$ & 112641.1 & -43 & 2117.5 & & \\
\hline $64(\mathrm{C})$ & 112638.9 & -43 & 2108.3 & & \\
\hline $65(\mathrm{D})$ & 112640.3 & -43 & 1817.6 & & \\
\hline $66(\mathrm{E})$ & 112649.6 & -43 & 1735.4 & & \\
\hline $67(\mathrm{~F})$ & 112652.7 & -43 & 1145.2 & & \\
\hline $68(\mathrm{G})$ & 112650.2 & -43 & 1135.9 & & \\
\hline $69(\mathrm{H})$ & 112458.5 & -43 & 1627.8 & & \\
\hline $76(\mathrm{P})$ & 112446.0 & -43 & 1231.7 & & \\
\hline $77(\mathrm{Q})$ & 112641.6 & -43 & 1527.1 & & \\
\hline $78(\mathrm{R})$ & 112455.7 & -43 & 0610.7 & & \\
\hline 1079 & 112629.1 & -43 & 0734.3 & & \\
\hline 1083 & 112615.5 & -43 & 0750.7 & & \\
\hline 1124 & 112600.8 & -43 & 0531.8 & & \\
\hline 2040 & 112632.7 & -43 & 1632.9 & & \\
\hline 2076 & 112621.2 & -43 & 2030.9 & & \\
\hline 2094 & 112546.3 & -43 & 2030.0 & & \\
\hline 2110 & 112610.2 & -43 & 2159.4 & & \\
\hline 2117 & 112633.8 & -43 & 2332.9 & & \\
\hline 2118 & 112627.0 & -43 & 2329.2 & & \\
\hline 2125 & 112613.0 & -43 & 2327.7 & & \\
\hline 3028 & 112501.3 & -43 & 1637.4 & & \\
\hline 3040 & 112500.8 & -43 & 1825.3 & & \\
\hline 3095 & 112458.6 & -43 & 2253.2 & & \\
\hline 3104 & 112519.7 & -43 & 2228.3 & & \\
\hline 3105 & 112519.8 & -43 & 2241.3 & & \\
\hline 3113 & 112536.9 & -43 & 2233.9 & & \\
\hline 4027 & 112447.6 & -43 & 1221.2 & & \\
\hline 4028 & 112448.3 & -43 & 1218.1 & & \\
\hline 4081 & 112451.1 & -43 & 0916.2 & & \\
\hline 4106 & 112526.8 & -43 & 0558.1 & & \\
\hline 4114 & 112511.6 & -43 & 0631.5 & & \\
\hline 4121 & 112453.0 & -43 & 0634.5 & & \\
\hline 4140 & 112536.5 & -43 & 0500.1 & & \\
\hline
\end{tabular}


quite uncrowded field, aperture photometry for aperture radii of 4, 5, 6, 7 and 8 pixels was derived for individual frames, using IRAF/APPHOT. The flat field errors were determined by the method described by Andersen et al. (1995b). Briefly, the magnitude differences for individual stars between offset and rotated exposures are used to determine a global fit to the flat field error. After applying this correction to the flat field, the 6-pixel aperture was identified as giving the best overall results, with a typical internal error of $0 . \mathrm{m}^{\mathrm{m}} 014$, independent of magnitude down to $V=15$ m. 5 .

Due to significant PSF variations across the field, the DAOPHOT package for PSF photometry (Stetson 1987) is not suitable as a general method. In a few cases, however, blended images of close doubles caused problems. For stars with faint, close companions (star pairs E30/30A, E47/47A, E59/59A, E21A/21B, and $6027 / 6028)$, the fainter stars were first subtracted with DAOPHOT/SUBSTAR. Aperture magnitudes were then redetermined from 3 frames. From the deduced magnitude offset to the previous aperture photometry, precise determinations of the contribution from the fainter components were made. Subsequently, the magnitudes of the fainter companions were determined by DAOPHOT/PSF photometry within each pair. In doing this, we only need to assume that PSF photometry is reliable over small areas where PSF variations are small.

The close bright pair 6027/6028 is a special case. Here, a large aperture (20 pixels radius) was used to determine the total magnitude of the pair relative to stars E32, E33, and E38, after subtracting all other stars from the frames. Their individual magnitudes were then derived, using the difference in PSF magnitude. Our final photometry in the instrumental system was formed as the average of the magnitudes measured from the individual images.

We have 29 stars in common with the photoelectric $u v b y-\mathrm{H}_{\beta}$ photometry of $\mathrm{N} 88$, two of which (E21A and E30) are now identified as visual binaries. The remaining 27 stars were used to derive the standard transformation. Since the observations were made in rapid succession and the standard stars are located in the same field, extinction correction can be simply incorporated in the colour transformation.

Using the procedure of Grønbech et al. (1976), we derived the following standard relations (see Fig. 1):

$$
\begin{gathered}
V_{\mathrm{std}}=y_{\mathrm{inst}}-0.089(b-y)_{\mathrm{inst}}+\mathrm{constant} \\
(b-y)_{\mathrm{std}}=0.955(b-y)_{\mathrm{inst}}+\text { constant }
\end{gathered}
$$

The reduced standard deviation in $\left(V_{\mathrm{NAA}}-V_{\mathrm{N} 88}\right)$ and $\left((b-y)_{\mathrm{NAA}}-(b-y)_{\mathrm{N} 88}\right)$ is $00^{\mathrm{m}} 0114$ and $00^{\mathrm{m}} 0044$ respectively. N88 lists standard errors as function of magnitude,

\footnotetext{
${ }^{1} \mathrm{IRAF}$ is developed and distributed by NOAO.
}
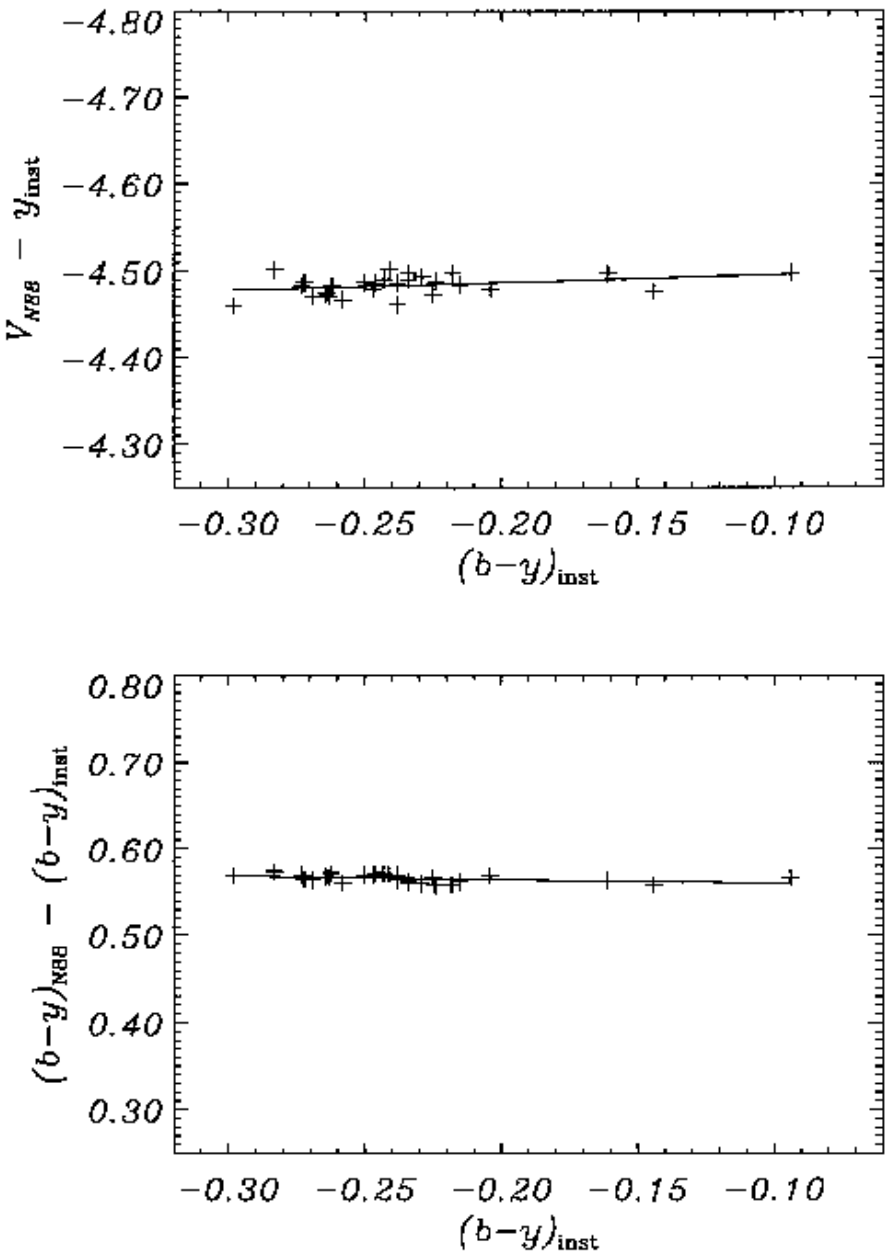

Fig. 1. a-b. Plots of the $V$ magnitude and $(b-y)$ colour transformations from our CCD instrumental system to N88, using the same scale as in Fig. 3

from which we deduce average standard errors of $0{ }^{\mathrm{m}} 0064$ and $0^{\mathrm{m}} 0047$ in $V$ and $(b-y)$ respectively. Assuming that the errors are uncorrelated, a standard error of 0.0094 in $V$ is derived for our data. In $(b-y)$ we are in the unusual, but statistically possible situation that the error derived from the comparison with N88 is smaller than the internal error in the $\mathrm{N} 88$ data. The standard errors in N88 are derived from repeated observations of $\approx 200$ stars and can therefore be considered as well determined. Given this, we can set an upper limit on our standard error in $(b-y)$ of 0.0034 at the $90 \%$ confidence level.

It is appropriate to note, however, that since the N88 photometry covers only the main-sequence and turnoff region of the CMD, our photometry cannot be claimed to have been properly standardised in the red-giant region of the CMD. The same comment presumably applies to the ATTS photometry. 
The final CMD for our full $13^{\prime} \times 13^{\prime}$ field surrounding NGC 3680, as constructed from our CCD photometry, is shown in Fig. 2.

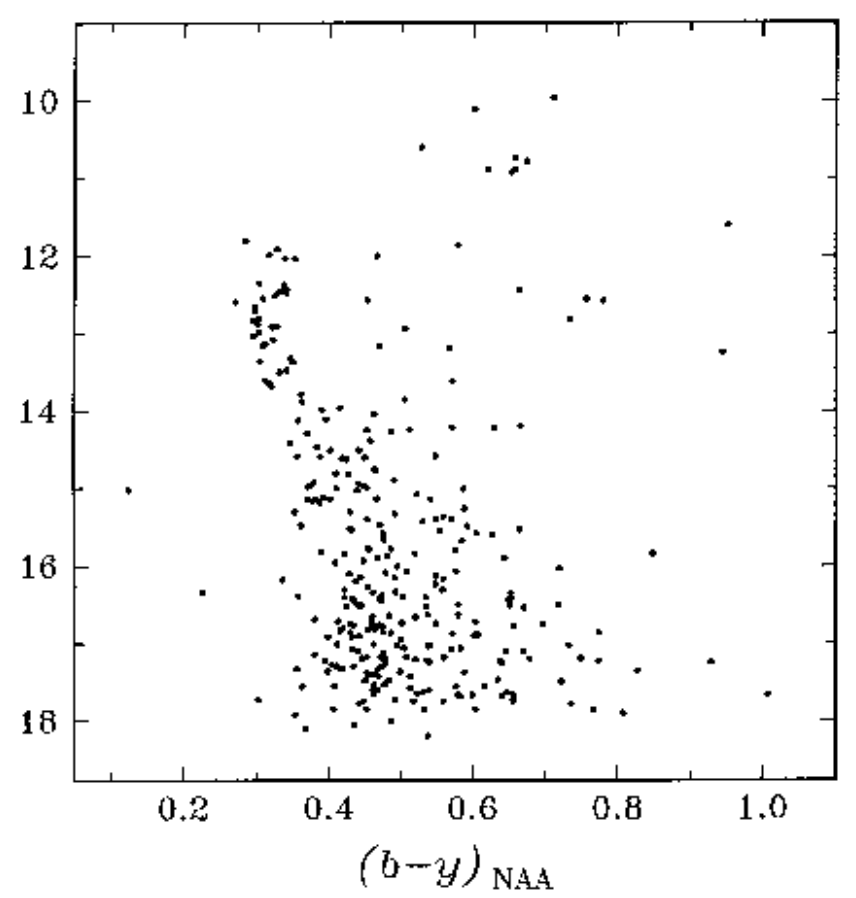

Fig. 2. Colour-magnitude diagram in the Strömgren system for all stars observed by us (NAA) in the field of NGC 3680

\subsection{Previous photometric data for NGC 3680}

We recall that the sources of $(U) B V$ photometry for NGC 3680 are Eggen (1969) and AHTC (photographic photometry calibrated by photoelectric and CCD standard observations). In the $u v b y-\mathrm{H}_{\beta}$ system, we have $\mathrm{N} 88$ (photoelectric photometry), ATTS (PSF-fitting CCD photometry), and the CCD aperture photometry reported above (NAA; $b$ and $y$ filters only). Since most theoretical isochrone calculations predict only broad-band colours as the directly observable parameters, it would be convenient to use the published $B V$ data. Before proceeding to do so, we have compared the photometry from all the above sources in order to detect any systematic trends as well as individual discrepancies for the stars in common between them.

Since both sets of uvby CCD photometry were calibrated using the stars in N88, it is to be expected that both $V$ and $(b-y)$ agree very well between those three papers, as functions of both magnitude and $(b-y)$ colour (Figs. 3 and 4). The exceptions are the close doubles E21A and E30 which, as mentioned above, were resolved by ATTS and by us, but not in the classical diaphragm photometry of N88, and E38 for which there seems to be a problem with the ATTS value of $m_{1}$. In addition, the ATTS value of $(b-y)$ for the red-giant binary E20, which

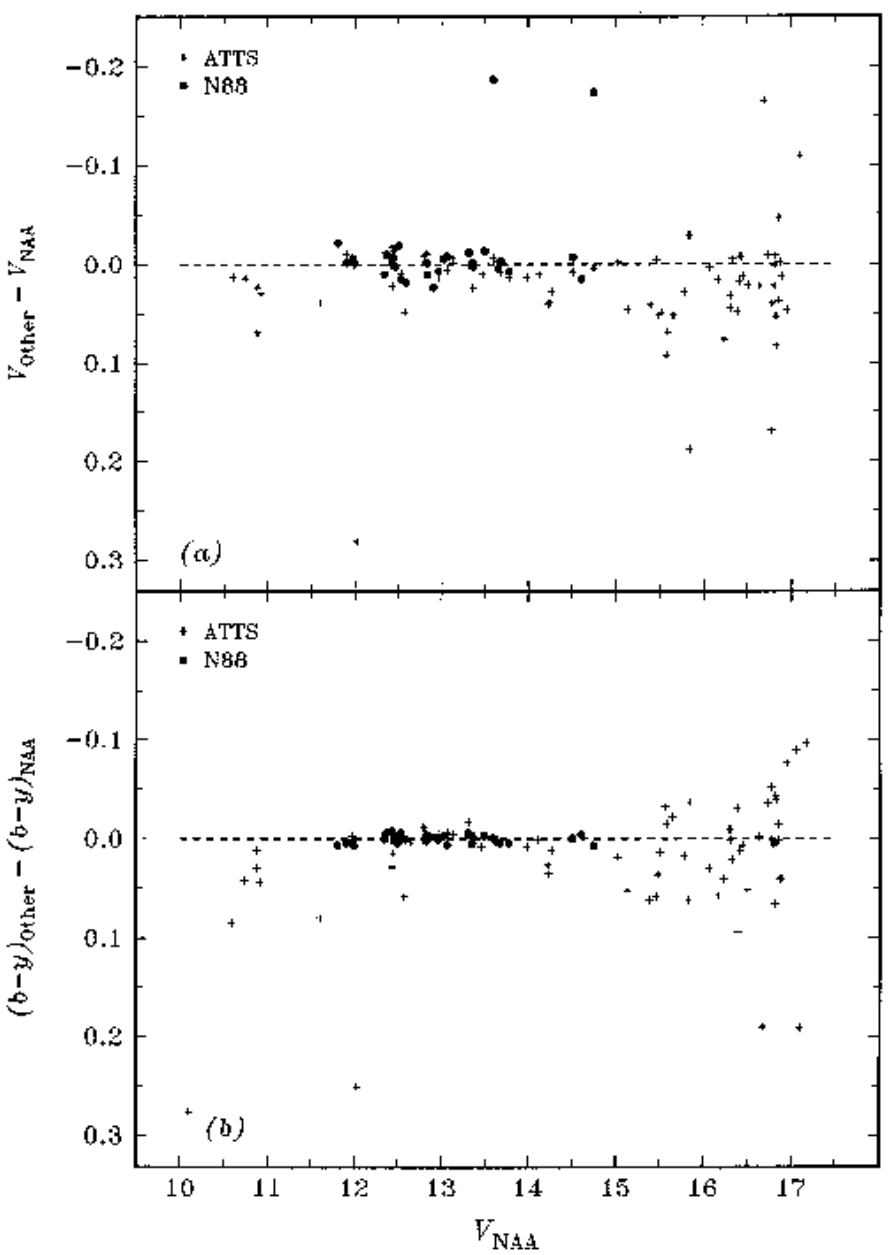

Fig. 3. $V$ magnitude and $(b-y)$ colour differences between our CCD data (NAA) and those by N88 (dots) and ATTS (plusses) vs. magnitude; the two discrepant stars from N88 are the close doubles E21A and E30

was not observed by N88, appears, for unknown reasons, to be some 0.20 too large. The rms differences (ATTSN88) for the remaining 17 stars in common are: $0.010(V)$, 0. $007(b-y), 0{ }^{\mathrm{m}} 009\left(m_{1}\right)$, and $0^{\mathrm{m}} 026\left(c_{1}\right)$. Except for $c_{1}$, these are in satisfactory agreement with the published error estimates. Our photometry and N88 were compared in Sect. 2.1.

In the $B V$ photometry for NGC 3680 we do, however, find significant systematic differences between the different sources, far exceeding the quoted mean errors. Figures 5-7 summarise the comparisons we have made. Omitting star E20, the AHTC $V$ magnitudes are systematically $00^{\mathrm{m}} 06$ fainter than those determined here (and by ATTS and N88) for the 248 stars in common, with an rms difference of 0.04 (Fig. 5). The Eggen $V$ magnitudes are on average 0.09 fainter than ours, with an rms dispersion of 0. 09 , based on 53 stars and omitting stars E17, I, P, and $\mathrm{R}$, which show very large discrepancies (Fig. 5). 


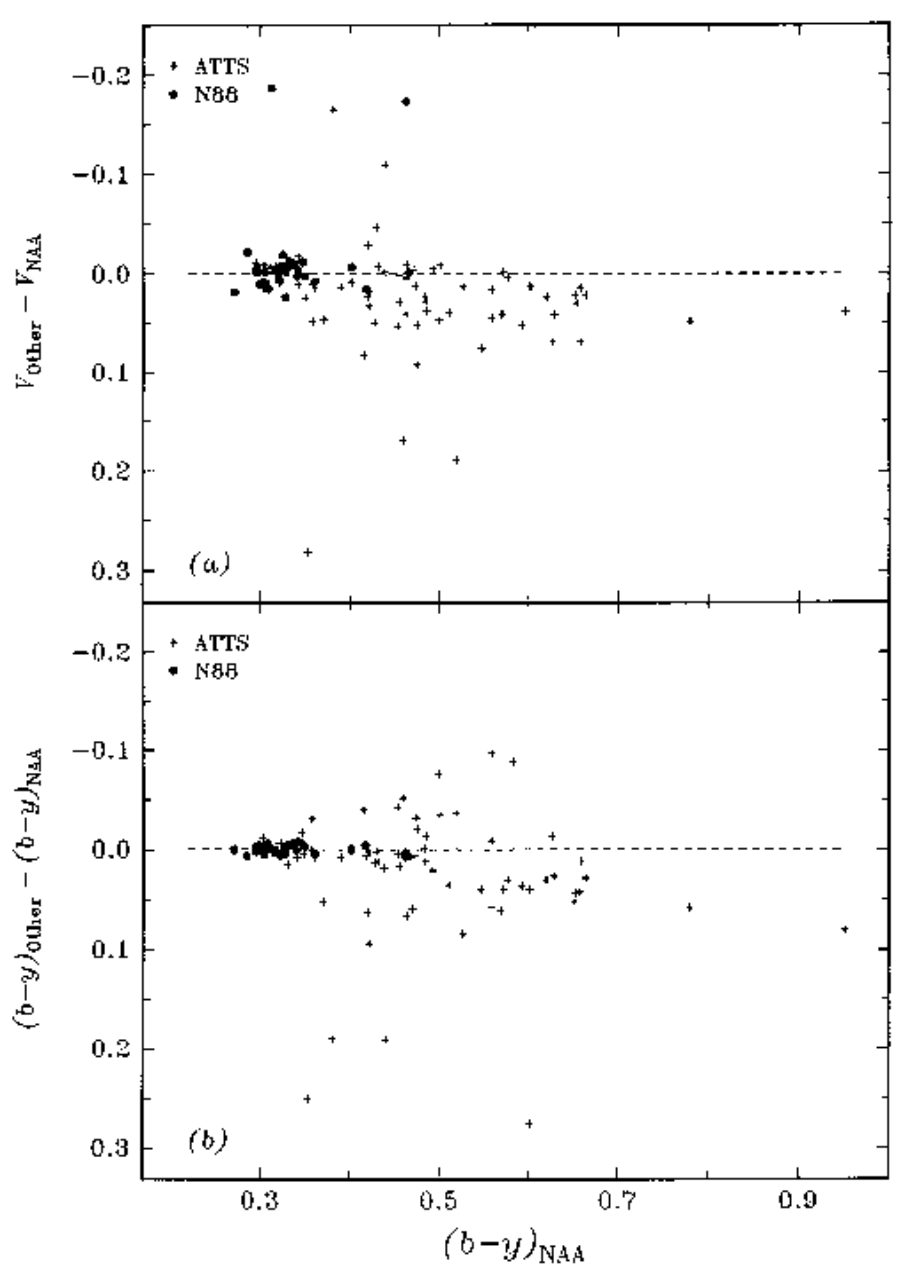

Fig. 4. $V$ magnitude and $(b-y)$ colour differences between our CCD data (NAA) and those by N88 (dots) and ATTS (plusses) vs. $(b-y)$ colour index

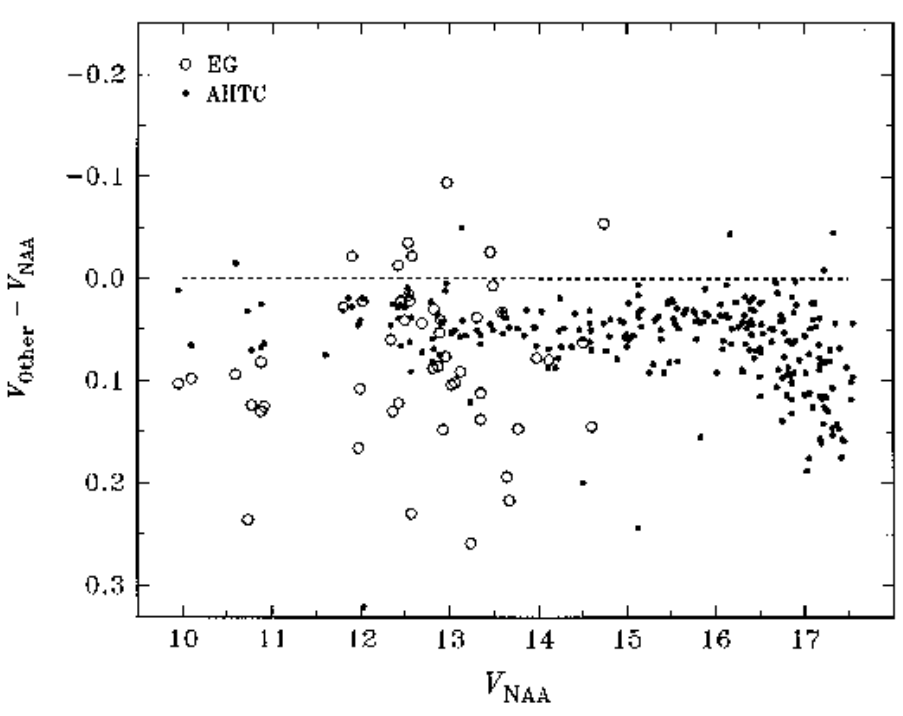

Fig. 5. $V$ magnitude differences between our data (NAA) and those by Eggen (circles) and AHTC (dots) vs. magnitude

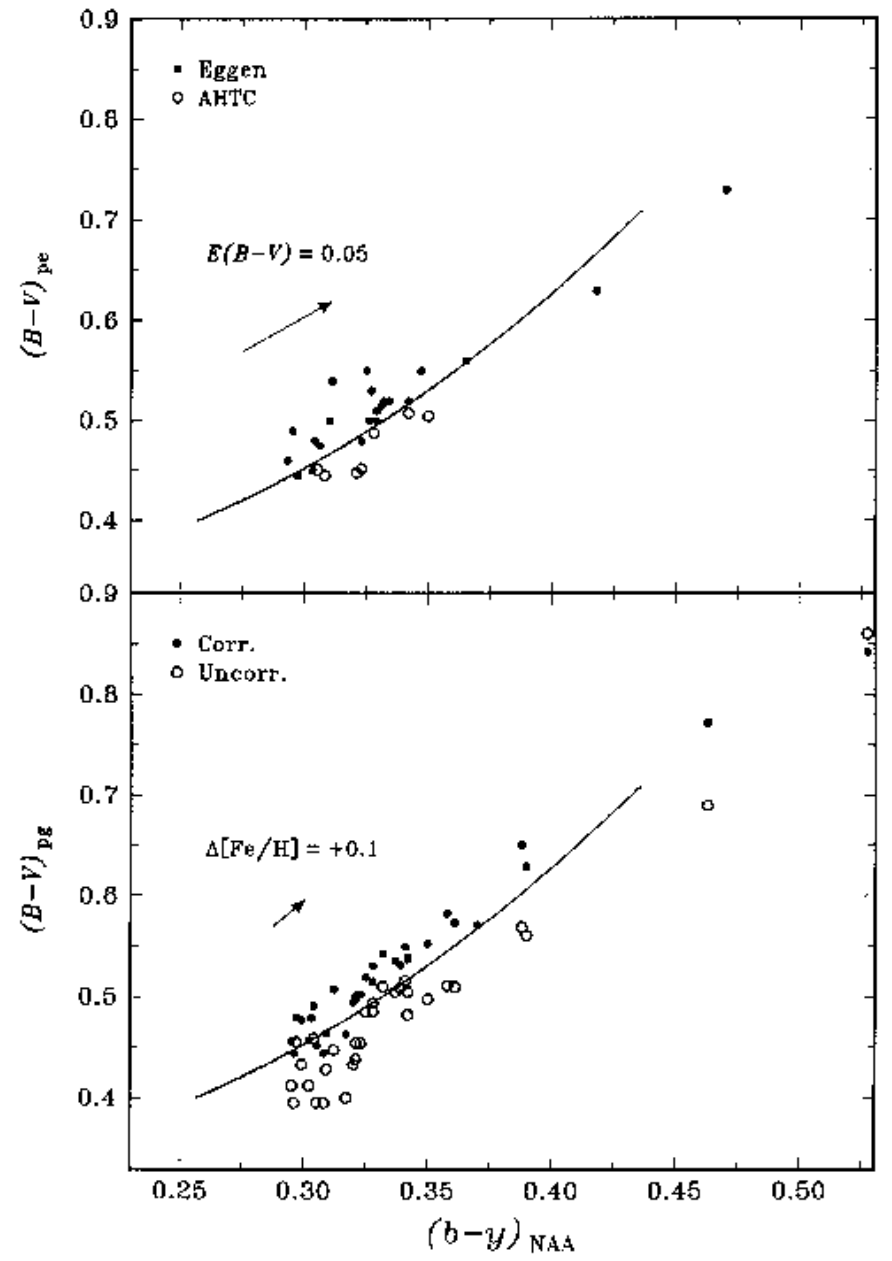

Fig. 6. Relation between the observed $(b-y)$ and $(B-V)$ colours for the radial-velocity members of NGC 3680. Upper: Photoelectric $(B-V)$ data. Lower: Photographic $(B-V)$ data from AHTC, both corrected as described in the text (dots) and directly as published (circles). The full line is the Hyades standard relation, reddened in both colours by the amounts observed for NGC 3680. Reddening and blanketing vectors appropriate to NGC 3680 are also shown

The systematic differences in $B-V$ between Eggen and AHTC, discussed extensively by AHTC, are illustrated in Fig. 7, which shows the difference between the (photoelectric) Eggen $(B-V)$ colours and the (photographic) $(B-V)_{\mathrm{AHTC}}$ data as published (circles). The large size and peculiar average trend of these differences as a function of $B-V$ itself led us to investigate the likely origin of these differences in more detail by plotting $(B-V)_{\mathrm{AHTC}}$ against $(b-y)$ for the main-sequence and turnoff stars in NGC 3680 (Fig. 6). Since the two clusters have the same metal abundance, the stars in NGC 3680 should fall on the Hyades relation, appropriately reddened in both colours. Accordingly, we plotted the Hyades standard main-sequence relation from Crawford \& Perry (1966) in the same diagram, reddened by $E(b-y)=$ 
0.036 as found for NGC 3680 (N88) and, correspondingly, $E(B-V)=0{ }^{\mathrm{m}} 050$.

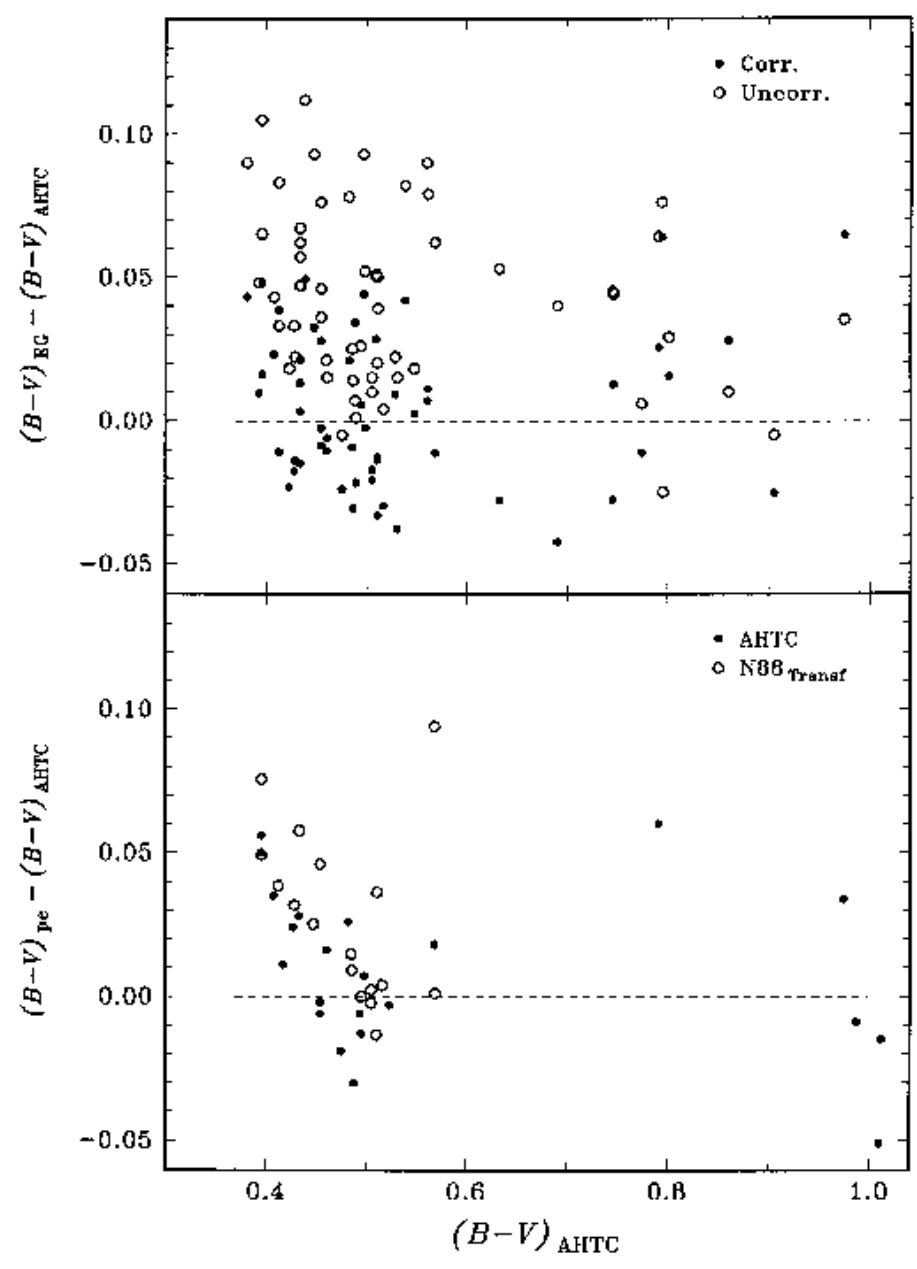

Fig. 7. Differences between the AHTC $(B-V)$ colours and other sources, vs. the published photographic $(B-V)_{\text {AнтC. }}$ Upper: Photoelectric $(B-V)$ data from Eggen minus $(B-V)_{\text {AHTC }}$, both corrected (dots) and as published (circles). Lower: Photoelectric $(B-V)$ values from AHTC (their Table 3 ; dots $)$ and from $(b-y)_{\mathrm{N} 88}$ as transformed to $(B-V)$ (circles), minus $(B-V)$ Антс, vs. $(B-V)$ Антс

Figure 6 shows that the expected agreement is manifestly not observed, neither for the photoelectric $(B-V)$ data by Eggen and later additional observations by AHTC (Fig. 6, upper panel), nor for the photographic data published by AHTC (lower panel). While most cluster stars near $(B-V)_{\mathrm{AHTC}}=0.5$ (circles) do lie near the reddened Hyades relation, most stars either redder or bluer than this have $(B-V)_{\mathrm{AHTC}}$ values that are some $0{ }^{\mathrm{m}} 04-0{ }^{\mathrm{m}} 05$ too blue for their $(b-y)_{\mathrm{N} 88}$. Reddening and blanketing vectors appropriate to NGC 3680 are shown in the upper and lower panels of Fig. 6 and indicate that errors in the assumed reddening or metal abundance of the cluster cannot cause the observed discrepancies. One is led to regard the published observational errors of the $B V$ photometry with considerable reservation.

Based on past experience, we assume that the N88 photometry for the individual uncrowded stars and thus the derived mean reddening and metallicity are substantially correct. The deviations from the Hyades relation seen in Fig. 6 would then be interpreted as a systematic error in the AHTC $B V$ photometry.

As a further check on this conjecture, we have drawn on such other photoelectric $(B-V)$ values as are available in addition to those by Eggen (1969). These are, first, the stars (re)observed by AHTC for calibration purposes (their Table 3; dots). Second, some independent values were obtained by transforming the N88 $(b-y)$ data to $(B-V)$ via the Hyades relation (Fig. 6), with appropriate reddening corrections. The differences of these transformed $(B-V)$ values from $(B-V)_{\text {AHTC }}$ have been added to the diagram in Fig. 7 (circles). As these independent and more recent photoelectric data show roughly the same form of systematic deviations from $(B-V)_{\mathrm{AHTC}}$ as the Eggen observations, it would again appear that their origin lies in the standardization problems encountered by AHTC.

We must conclude that the published $B V$ photometry for NGC 3680 is not accurate enough for our main purpose, a precise observational test of theoretical isochrone calculations. The strong deviation of the bluest mainsequence stars from the Hyades relation is particularly disconcerting, since these stars define the turnoff point, while the main-sequence termination point occurs near $(B-V)_{\text {AHTC }}=0.5$, where the stars do fall closer to the Hyades relation. Thus, not only does the turnoff colour of NGC 3680 (hence, its age) become uncertain, but the entire shape of the turnoff itself, one of the key diagnostics of the computed isochrones, becomes strongly distorted.

This conclusion, combined with the lack of uvby data for many of the stars in the field, led us to redetermine the CMD of NGC 3680 in the uvby system as reported above, and the comparisons with theoretical isochrones in Paper II will be made primarily using these data.

However, in response to a draft of this paper, Drs. Anthony-Twarog and Twarog kindly re-examined the calibration of their photographic observations in considerable detail. They found that, apart from a small offset (see Fig. 5), their $V$ magnitude scale is substantially correct as published, certainly so for stars brighter than $V$ $=15.5$. However, the $B$ magnitude calibration turns out to contain a non-linearity which the previously available body of calibration stars had been inadequate to reveal. This non-linearity propagates directly into the $(B-V)$ colours since magnitude and colour are related for the cluster stars. Drs. Anthony-Twarog and Twarog recommend the following correction formula for their published photographic $(B-V)$ colours: 
$(B-V)_{\mathrm{corr}}=0.98(B-V)_{\mathrm{AHTC}}+0.023 V_{\mathrm{AHTC}}-0.244$

The corrected $(B-V)$ values are shown (as dots) alongside the published data in Figs. 6 (lower panel) and 7 (upper panel). The agreement with the Hyades relation, and especially with the Eggen $(B-V)$ colours, is clearly improved. However, systematic and random uncertainties of a few hundredths of a magnitude appear to remain, more than desirable for the detailed examination of the shape of the turnoff region of the cluster sequence which is the primary objective of this study (see Paper II).

Still, for consistency checks, for the red giants for which the by photometry cannot be properly calibrated using the F stars from N88, and when stars outside the CCD field are considered, we will occasionally make use of the AHTC $B V$ photometry, and then with the corrections described above.

\section{Radial-velocity data for NGC 3680}

\subsection{Observations}

Radial-velocity observations of NGC 3680 were made with the photoelectric scanner CORAVEL, attached to the Danish 1.5-m telescope at ESO, La Silla, during several observing periods from January 1988 through January 1994. CORAVEL, its accuracy and radial-velocity zeropoint are discussed by Baranne et al. (1979), Mayor (1985) and Mayor \& Maurice (1985). The observing list contained all 118 stars in the AHTC field around NGC 3680 which were potentially observable with the fixed K2 III mask of CORAVEL, i.e. all stars brighter than $B \approx 15.2$ and redder than $B-V \approx 0.38$. The red giants in NGC 3680 were also observed in order to define the mean radial velocity and velocity dispersion of NGC 3680; these data were recently published together with those on the red giants in the "sister cluster" of NGC 3680, IC 4651 (Mermilliod et al. 1995) and will, therefore, not be discussed further here. The present study is directed primarily at the main sequence and turnoff regions of NGC 3680, which are the crucial features of the CMD for determining the distance and age of the cluster, and for testing stellar models with different convection prescriptions.

Our observing strategy was designed with the primary aim to define a clean sample of single member stars in NGC 3680 from among the binaries and/or nonmembers. To this end, we have attempted to secure four or more observations, distributed over at least three years, for all candidate radial-velocity and/or photometric members of NGC 3680. A CMD of the complete sample of stars observed in the field of NGC 3680 is shown in Fig. 8 ( $B V$ data from AHTC).

Due to the difficulty of locating individual binary components correctly in the CMD, even if membership is supported by a systemic velocity derived from a full orbital

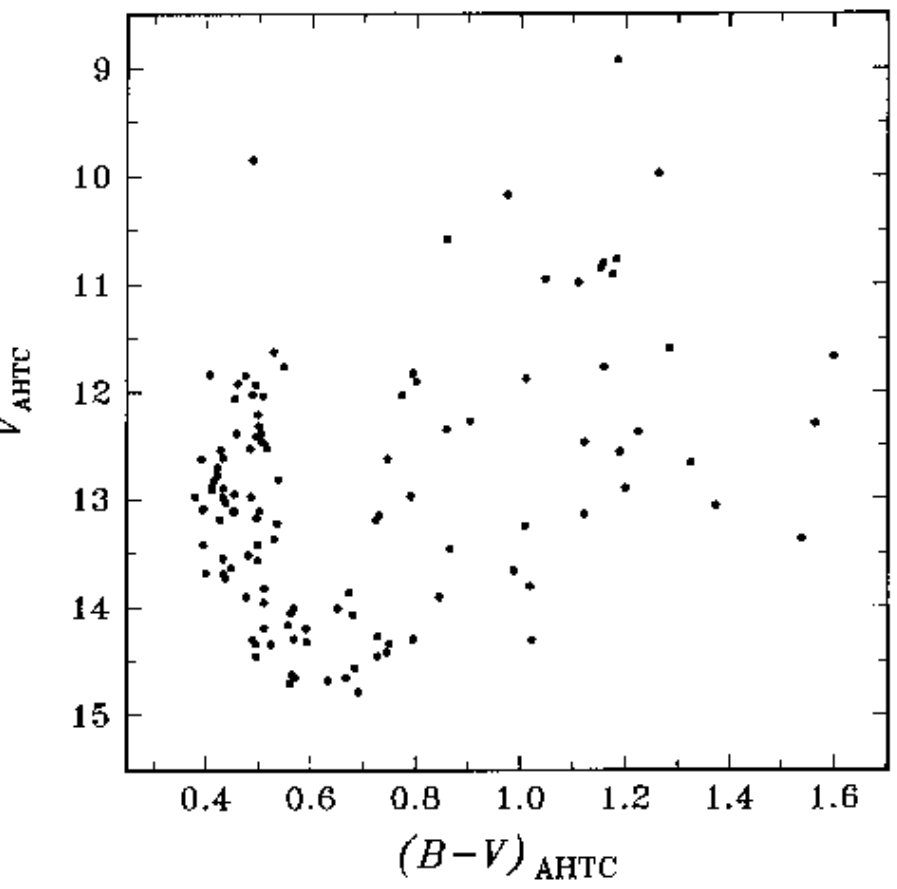

Fig. 8. $B V$ colour-magnitude diagram (AHTC data) of all stars observed with CORAVEL in the AHTC field around NGC 3680

solution, we have not at this stage pursued the observation of suspected binary stars much beyond the point of unambiguous variability detection. Similarly, if two concordant observations classified a star as a non-member, we have not expended inordinate extra effort in a search for possible low-level variability. In all, about 400 observations were obtained on the program from January 1988 through January 1994.

\subsection{Data reduction}

A note on the reduction of the measurements is in order, because the important turnoff region in NGC 3680 contains stars that are near the upper limits in both temperature and rotation for stars to be observable with CORAVEL. At the same time, unambiguous separation of cluster and field stars places strict demands on the accuracy of the observed radial velocities.

As detailed by Baranne et al. (1979) and Mayor (1985), the cross-correlation profile is recorded directly in 40 or 64 points by CORAVEL. The radial velocity is determined by fitting the position, depth, and width of a Gaussian profile plus a continuum level to these data. For a single, constant star, the profile parameters derived from a single observation are more or less precise estimates of the true values, depending on the (photon) noise level of each data set. For observations with count levels significantly below average the fitted profile may deviate drastically from the rest, especially for fast-rotating stars. In these cases, it 
is preferable to fix the profile shape parameters to their weighted mean value over all well-integrated observations of that star, leaving only the peak position (i.e. radial velocity) and continuum level as free parameters in the fit. Similarly, in double-lined binaries, the profile shapes are fixed to their mean values from those observations where the profiles are resolved.

\subsection{Rotational velocities}

From the width of the observed Gaussian cross-correlation profiles, rotational velocities can be computed following Benz \& Mayor $(1981,1984)$. Thus, mean rotational velocities ( $v \sin i$, with errors) have been determined for all the programme stars. For some sharp-lined stars only an upper limit to $v \sin i$ could be determined, due to the finite $S / N$ of the profiles; no error estimate is then given. Because CORAVEL is optimized for observations of sharplined, late-type stars, both rotational and radial velocities become relatively uncertain for $v \sin i>30-40 \mathrm{~km} \mathrm{~s}^{-1}$.

\subsection{Results}

Altogether, some 400 new radial-velocity observations have been obtained of 118 main-sequence, turnoff, and field stars in the $20^{\prime} \times 20^{\prime}$ field around NGC 3680 for which photometry was obtained by AHTC. Table 2 lists weighted mean radial and rotational velocities, with standard errors, for 109 of these stars, determined as described above, together with additional data on the observations and notes on duplicity, etc. A few stars were found to be too hot and/or too fast-rotating to yield a measurable cross-correlation signal with CORAVEL, viz., E42, E55, 69(H), 72(K), 78(R), and AHTC 1094, 2030, 2110, and 6027 (ATTS 2027). For completeness, together with the new rotational data, we include the red-giant radial velocities from Mermilliod et al. (1995), identified by remark "M" in Table 2. The individual radial-velocity observations of the new stars, some of which might still turn out to be binaries if monitored over a long period, are given in Table 3 (only available in electronic form, see footnote at the beginning of the paper).

A histogram of the observed radial velocities (Fig. 9) leaves no doubt as to the existence of a real concentration of stars near the mean velocity of NGC 3680 itself, $0.87 \mathrm{~km} \mathrm{~s}^{-1}$ as determined from the six constant-velocity red giants (Mermilliod et al. 1995). It also illustrates, however, that of the order of 8 field stars can still be expected in the $5 \mathrm{~km} \mathrm{~s}^{-1}$ velocity bin centered on the cluster. The detailed identification of these stars will be discussed in Paper II.

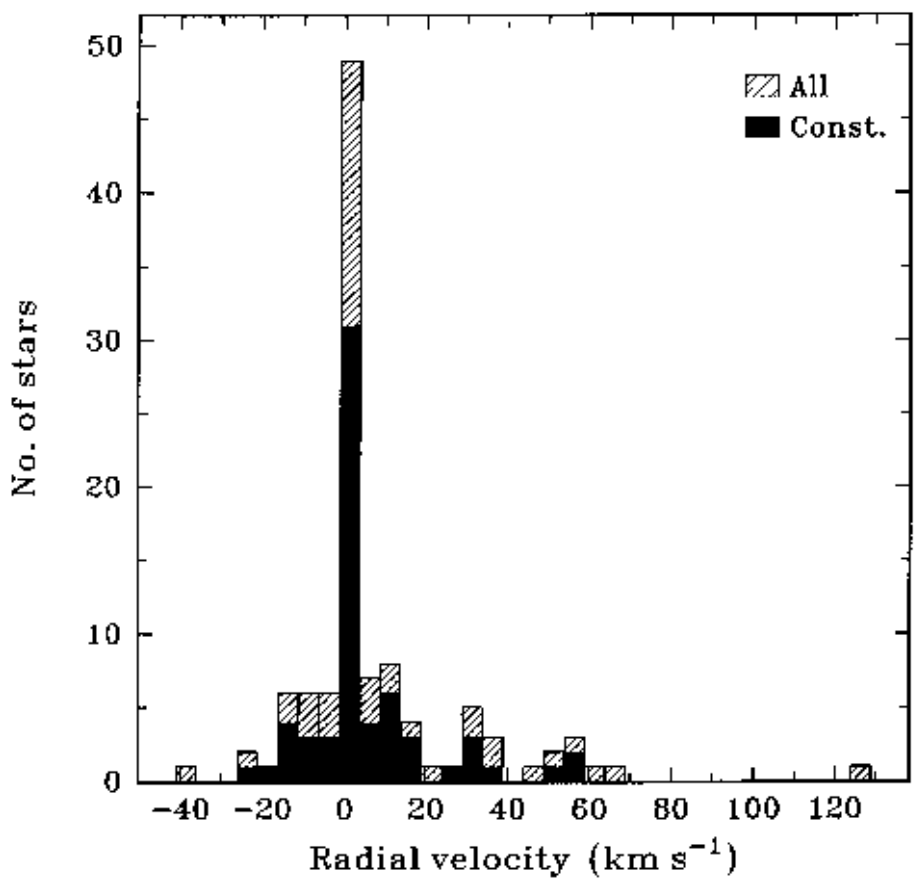

Fig. 9. Histogram of the observed radial velocities from Table 2 , highlighting the stars judged to be constant from two or more observations

\subsection{Notes on individual stars}

Asterisks in Table 2 identify stars on which separate comments are given in the following (see also Mermilliod et al. 1995 for specific comments on the red giants):

E1 is a double-lined binary with a large luminosity ratio; the secondary has $v \sin i<10 \mathrm{~km} \mathrm{~s}^{-1}$.

E11, a red giant, shows velocity variations at a low level, but with high significance. It is some 0.05 bluer in $(B-V)$ than the clump and probably has an upper main-sequence companion.

E14 is a spectroscopic binary with components of slightly different luminosity; the secondary has $v \sin i=$ $10 \pm 15 \mathrm{~km} \mathrm{~s}^{-1}$.

E18 is a short-period single-lined spectroscopic binary and non-member (Mermilliod et al. 1995).

E20 and 34 were noted as probable giant binaries by AHTC, being much brighter and bluer than the other clump stars. Both are radial-velocity members and E20 indeed a textbook double-lined binary (Mermilliod et al. 1995). Surprisingly, ATTS found E20 to be far redder than the clump and a probable photometric nonmember, suggesting a problem with their CCD data for this star.

E21 is an F star with $(b-y)=0.40$ from both ATTS, N88, and our data, and a normal CORAVEL profile for that colour. AHTC give $(B-V)=2.35$, which must be a misprint.

E21A and 30 are close double stars. They were omitted by AHTC, and the N88 uvby photometry refers to the 
combined light. The stars are resolved in PSF-fitting photometry, as noted in Sect. 2.1. Removing the light of the companions moves these stars downwards and to the blue in the cluster CMD relative to the positions shown by N88.

E22 is a double-lined binary with near-equal components; the secondary has $v \sin i=10.6 \pm 2.9 \mathrm{~km} \mathrm{~s}^{-1}$.

E27, a red giant, is an unambiguous velocity variable, but is located precisely together with the single clump giants. A faint, late-type main-sequence companion would be consistent with these observations.

The red giant E34 in the center of NGC 3680, although not significantly variable in velocity during the 10 -year period covered by the CORAVEL observations, has a crosscorrelation profile only about half as deep as the other cluster giants. As it is also significantly bluer than the bulk of the red giants, we conclude that it has a blue, upper main-sequence or turnoff companion in a long-period and/or low-inclination orbit.

E36 is a double-lined binary; the secondary has $v \sin i=$ $9.1 \pm 3 \mathrm{~km} \mathrm{~s}^{-1}$ (estimated error for both components).

E38 was considered metal-poor by ATTS on the basis of its $m_{1}$ index, but normal by N88 and AHTC. We include it in our sample of normal stars.

The red giant E41 was found by ATTS to have an anomalous $m_{1}$ index and be a likely non-member. It has constant radial velocity equal to the cluster mean, and a cross-correlation profile and $(B-V)$ index just like the other clump giants. Given no other evidence of peculiarity, we consider it to be a normal, single cluster member.

E62(A) is a double-lined binary with a sharp-lined primary; the secondary has $v \sin i=22.5 \pm 3.4 \mathrm{~km} \mathrm{~s}^{-1}$.

4094, the single photometric lower giant-branch candidate, is a marginal radial-velocity member. As discussed by AHTC, 4094 may be the star actually observed by Eggen (1969) under the name of E17, rather than the star so identified on his chart.

6016 (ATTS 2016) is one of two stars identified as AHTC 3001 in ATTS. Consistent with the AHTC chart, we have retained the number 3001 for the brighter of the two stars close to the central red giant E34, while 6016 is its faint close companion.

$6027 / 6028$ (ATTS 2027/2028) is another close pair resolved by ATTS and us, and observable separately with CORAVEL in good seeing. For 6027, which has a fairly red $(b-y)$ colour and unusual $m_{1}$ index in the ATTS photometry, we find a much bluer $(b-y)$ which may explain why (like E55) it shows no correlation peak with CORAVEL. 6028 is confirmed as a (double-lined) cluster binary as predicted by AHTC; the secondary component has $v \sin i=$ $5.5 \mathrm{~km} \mathrm{~s}^{-1}$ (upper limit).

\section{Spectroscopic binary detection}

From each observed CORAVEL profile, the predicted standard error can be computed. This error estimate $\left(\sigma_{\mathrm{C}}\right)$ is a function of the observed count level, profile shape, and integration time (scintillation noise) as described in more detail by Mayor (1985), Andersen et al. (1985), and Duquennoy \& Mayor (1991).

From the observed and computed errors, we can compute the value of $\chi^{2}$ for each star and the probability $P\left(\chi^{2}\right)$ that it could arise purely due to random errors. When that probability is less than $1 \%$, the star is classified as a probable binary; otherwise, the default hypothesis of a constant-velocity, single star is retained. Stars found to be constant on the basis of two or more observations and on this criterion are highlighted in Fig. 9.

For reliable results, this procedure clearly requires that $\sigma_{\mathrm{C}}$ is correctly estimated. If this condition is satisfied, the ratio $O / C$ between observed standard deviation $\left(\sigma_{\mathrm{E}}\right)$ and computed error $\left(\sigma_{\mathrm{C}}\right.$, mean value) should average unity for constant stars, regardless of, e.g. rotation. However, in early F-type stars such as the turnoff stars in NGC 3680, rotational broadening is in fact the most serious impediment to obtaining accurate radial velocities: Indeed, no useful data at all could be obtained for a few stars, as noted above (Sect. 3.4). As the profile width enters significantly in the calculation of the expected error, but is poorly determined in very shallow profiles, it is important to check whether our variability criterion is adequate and consistent for all rotations.

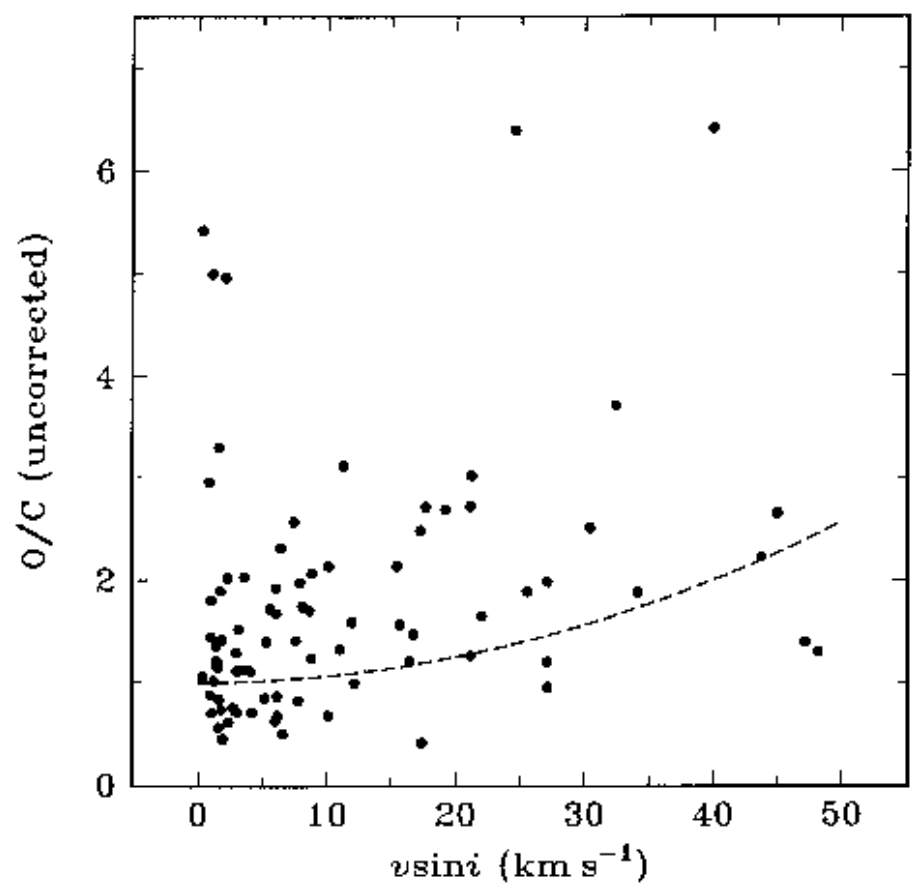

Fig. 10. The ratio of observed $(O)$ to computed $(C)$ velocity errors from the standard CORAVEL reductions vs. rotational velocity. Note the apparent absence of constant stars $(O / C$ $<1)$ for $v \sin i>15 \mathrm{~km} \mathrm{~s}^{-1}$. The dashed curve indicates our approximate correction factor to the computed error estimates. A few large $O / C$ values are off scale 
Table 2. Mean CORAVEL radial and rotational velocities $\left(\mathrm{km} \mathrm{s}^{-1}\right)$ and derived parameters for 109 stars in the AHTC field of $20^{\prime} \times 20^{\prime}$ around NGC 3680. The remark "M" refers to data from Mermilliod et al. (1995), an asterisk to Sect. 3.5

\begin{tabular}{|c|c|c|c|c|c|c|c|c|c|c|c|c|c|}
\hline Id no. & K no. & $<R V>$ & $\sigma_{<R V>}$ & $n$ & $\sigma_{\mathrm{E}}$ & $\sigma_{\mathrm{C}}$ & $O / C$ & $P\left(\chi^{2}\right)$ & $\Delta R V$ & $\Delta T$ & $v \sin i$ & $\sigma_{v \sin i}$ & Remarks \\
\hline 1 & 1035 & -4.96 & 2.10 & 5 & 13.30 & 2.71 & 3.21 & 0.000 & 35.9 & 763 & 40.0 & 10.0 & $*$ \\
\hline 2 & 1076 & -8.27 & 0.31 & 3 & 0.35 & 0.52 & 0.66 & 0.661 & 0.9 & 420 & 6.2 & 4.5 & \\
\hline 3 & 3006 & 11.86 & 0.36 & 5 & 0.81 & 0.47 & 1.68 & 0.024 & 2.0 & 2305 & 5.6 & 1.5 & \\
\hline 4 & 1083 & 0.91 & 0.63 & 5 & 0.49 & 1.18 & 0.35 & 0.976 & 1.1 & 1968 & 17.4 & 2.5 & \\
\hline 5 & 1194 & -1.50 & 6.76 & 8 & 19.12 & 0.57 & 32.09 & 0.000 & 48.4 & 1591 & 7.6 & 1.7 & \\
\hline 6 & 1207 & 0.37 & 0.52 & 5 & 0.96 & 0.80 & 0.83 & 0.622 & 2.2 & 1941 & 27.1 & 1.2 & \\
\hline 7 & 1337 & 57.67 & 1.05 & 2 & 1.49 & 0.50 & 2.96 & 0.003 & 2.1 & 308 & 0.9 & 5.5 & \\
\hline 8 & 1321 & 9.40 & 0.23 & 4 & 0.47 & 0.36 & 1.28 & 0.184 & 1.3 & 668 & 3.0 & 2.0 & \\
\hline 9 & 1327 & 2.59 & 0.50 & 5 & 1.11 & 0.54 & 1.97 & 0.004 & 2.6 & 811 & 8.9 & 1.7 & \\
\hline 10 & 1319 & -1.01 & 1.16 & 8 & 1.73 & 1.34 & 0.53 & 0.968 & 6.3 & 1944 & 48.2 & 16.9 & \\
\hline 11 & 1286 & -0.27 & 0.20 & 8 & 0.57 & 0.30 & 1.89 & 0.001 & 1.7 & 3657 & 1.7 & 1.3 & $\mathrm{M}^{*}$ \\
\hline 13 & 1175 & 0.90 & 0.12 & 7 & 0.31 & 0.30 & 1.01 & 0.410 & 0.9 & 2981 & 1.2 & 1.6 & $\mathrm{M}$ \\
\hline 14 & 1168 & 0.83 & 0.73 & 12 & 4.06 & 1.35 & 4.65 & 0.000 & 44.4 & 1565 & 24.5 & 12.8 & * \\
\hline 15 & 1192 & 1.93 & 0.38 & 7 & 1.01 & 0.64 & 1.36 & 0.107 & 3.9 & 1541 & 15.7 & 1.1 & \\
\hline 16 & 1197 & 37.87 & 7.11 & 6 & 17.42 & 0.90 & 18.64 & 0.000 & 42.5 & 1493 & 8.0 & 2.4 & \\
\hline 17 & 1116 & 31.46 & 0.19 & 3 & 0.24 & 0.33 & 0.73 & 0.594 & 0.5 & 736 & 2.9 & & $\mathrm{M}$ \\
\hline 18 & 1176 & -2.34 & 0.07 & 41 & 0.36 & 0.34 & 30.75 & 0.000 & 33.9 & 3000 & 2.3 & 0.8 & $\mathrm{M}^{*}$ \\
\hline 19 & 1208 & 0.41 & 1.14 & 7 & 3.01 & 1.60 & 1.09 & 0.372 & 9.8 & 1242 & 34.1 & & \\
\hline 20 & 1261 & 0.62 & 0.13 & 14 & 1.04 & 0.30 & 9.99 & 0.000 & 12.8 & 3769 & 1.3 & 1.2 & $\mathrm{M}^{*}$ \\
\hline 21 & 1306 & 11.04 & 0.36 & 4 & 0.72 & 0.47 & 1.51 & 0.081 & 1.8 & 421 & 3.2 & & $*$ \\
\hline $21 \mathrm{~A}$ & 1304 & 1.58 & 0.57 & 4 & 1.14 & 0.44 & 2.48 & 0.000 & 2.4 & 1487 & 7.4 & 2.9 & $*$ \\
\hline 22 & 1318 & 1.32 & 0.26 & 11 & 1.28 & 0.90 & 35.23 & 0.000 & 84.3 & 1857 & 6.1 & 3.1 & * \\
\hline 23 & 1310 & 0.67 & 0.58 & 6 & 1.42 & 0.43 & 3.29 & 0.000 & 4.2 & 1487 & 1.6 & 2.6 & \\
\hline 24 & 1330 & 2.44 & 0.57 & 5 & 1.27 & 0.66 & 1.88 & 0.009 & 2.9 & 1968 & 6.1 & 3.0 & \\
\hline 25 & 1348 & 2.15 & 0.61 & 7 & 1.61 & 0.75 & 2.01 & 0.001 & 4.0 & 1970 & 10.2 & 1.9 & \\
\hline $25 \mathrm{~A}$ & 1331 & -0.75 & 1.04 & 5 & 2.32 & 1.17 & 1.36 & 0.130 & 6.0 & 1215 & 27.1 & 2.1 & \\
\hline 26 & 1374 & 0.32 & 0.11 & 8 & 0.31 & 0.29 & 1.06 & 0.358 & 0.9 & 3657 & 0.3 & 1.7 & M \\
\hline 27 & 1404 & 0.83 & 0.46 & 10 & 1.45 & 0.29 & 4.99 & 0.000 & 4.5 & 3769 & 1.1 & & $\mathrm{M}^{*}$ \\
\hline 29 & 1417 & 3.44 & 0.18 & 7 & 0.37 & 0.46 & 0.79 & 0.728 & 1.2 & 1966 & 7.8 & 1.3 & \\
\hline 30 & 1396 & 1.57 & 0.90 & 5 & 1.94 & 0.91 & 1.86 & 0.011 & 4.8 & 1616 & 15.5 & 2.3 & $*$ \\
\hline 31 & 1405 & 8.24 & 1.01 & 4 & 2.03 & 0.76 & 2.18 & 0.003 & 4.3 & 671 & 19.2 & 1.9 & \\
\hline 32 & 1454 & 1.37 & 0.98 & 5 & 2.20 & 0.81 & 2.27 & 0.001 & 5.5 & 1140 & 17.7 & 1.5 & \\
\hline 33 & 1350 & -0.93 & 1.89 & 9 & 5.67 & 0.60 & 8.16 & 0.000 & 14.1 & 1967 & 16.1 & 0.9 & \\
\hline 34 & 1379 & 1.29 & 0.15 & 8 & 0.44 & 0.32 & 1.36 & 0.082 & 1.5 & 3297 & 1.4 & 1.6 & $\mathrm{M}^{*}$ \\
\hline 35 & 1376 & 0.61 & 0.33 & 5 & 0.66 & 0.67 & 0.91 & 0.525 & 1.6 & 1615 & 12.1 & 1.4 & \\
\hline 36 & 1381 & 1.12 & 0.47 & 5 & 1.78 & 0.86 & 9.63 & 0.000 & 31.2 & 744 & 11.9 & & $*$ \\
\hline 37 & 1395 & 2.18 & 0.76 & 7 & 2.01 & 1.06 & 1.34 & 0.116 & 6.5 & 1149 & 25.5 & 1.7 & \\
\hline 38 & 1347 & 1.11 & 2.02 & 5 & 4.51 & 2.03 & 1.01 & 0.432 & 12.0 & 1541 & 43.7 & 4.7 & $*$ \\
\hline 39 & 1324 & 1.27 & 0.41 & 6 & 1.00 & 0.50 & 1.90 & 0.003 & 2.4 & 2284 & 7.9 & 8.4 & \\
\hline 40 & 1334 & -15.26 & 0.19 & 4 & 0.39 & 0.35 & 1.10 & 0.308 & 0.9 & 669 & 3.0 & & \\
\hline 41 & 1461 & 0.93 & 0.13 & 6 & 0.33 & 0.29 & 1.15 & 0.256 & 1.0 & 2982 & 1.5 & & $\mathrm{M}^{*}$ \\
\hline 43 & 1510 & 2.71 & 0.80 & 6 & 1.93 & 1.19 & 1.27 & 0.183 & 4.9 & 2284 & 22.0 & 4.0 & \\
\hline 44 & 1469 & 1.15 & 0.10 & 7 & 0.19 & 0.27 & 0.70 & 0.816 & 0.6 & 2962 & 1.1 & 1.4 & M \\
\hline 45 & 1522 & 1.60 & 0.48 & 5 & 1.06 & 0.84 & 0.99 & 0.435 & 2.4 & 1476 & 21.1 & 2.2 & \\
\hline 46 & 1507 & 2.00 & 0.46 & 5 & 1.04 & 0.78 & 1.24 & 0.198 & 2.9 & 1970 & 11.0 & 2.2 & \\
\hline $46 \mathrm{~A}$ & 1524 & 7.43 & 1.64 & 5 & 3.67 & 1.46 & 1.59 & 0.041 & 9.4 & 835 & 30.4 & & \\
\hline 48 & 1640 & -14.02 & 3.22 & 2 & 4.56 & 1.72 & 1.17 & 0.245 & 6.6 & 1546 & 45.0 & 5.0 & \\
\hline 49 & 1653 & 67.45 & 9.37 & 4 & 18.74 & 0.56 & 32.41 & 0.000 & 43.2 & 663 & 7.9 & 2.1 & \\
\hline 50 & 1781 & 50.70 & 0.18 & 7 & 0.48 & 0.40 & 1.21 & 0.213 & 51.3 & 2924 & 1.4 & & M \\
\hline 53 & 1873 & 0.64 & 0.12 & 7 & 0.23 & 0.32 & 0.73 & 0.782 & 0.8 & 2962 & 1.8 & 1.5 & $\mathrm{M}$ \\
\hline 54 & 1899 & 16.54 & 0.50 & 7 & 1.31 & 0.24 & 5.42 & 0.000 & 3.2 & 2962 & 0.3 & & $\mathrm{M}$ \\
\hline 57 & 1661 & 33.85 & 4.04 & 2 & 3.33 & 2.39 & 0.58 & 0.566 & 5.0 & 62 & 47.1 & 7.2 & \\
\hline 58 & 1638 & 34.01 & 19.28 & 3 & 33.39 & 0.79 & 38.49 & 0.000 & 64.8 & 363 & 12.7 & 2.5 & \\
\hline 59 & 1643 & -13.67 & 0.26 & 3 & 0.31 & 0.44 & 0.70 & 0.613 & 0.6 & 741 & 4.2 & 3.0 & \\
\hline
\end{tabular}

We have tested this by plotting the individual, raw values of $O / C$ as a function of $v \sin i$ (Fig. 10). For sharplined stars, one does observe the expected clustering of stars around the value $O / C=1$, with a scattering of larger $O / C$ values indicating true variability. For $v \sin i>15-$ $20 \mathrm{~km} \mathrm{~s}^{-1}$, however, the sample appears to consist exclusively of definite, but low-amplitude variables, an unlikely situation. We conclude that the standard CORAVEL reductions somewhat underestimate the velocity errors for rotating stars.

As a suitable, smooth correction function rising relatively steeply with $v \sin i$ as indicated by Fig. 10, we have chosen to "correct" the predicted errrors of a single velocity as computed by the standard CORAVEL reduction 
Table 2. continued

\begin{tabular}{|c|c|c|c|c|c|c|c|c|c|c|c|c|c|}
\hline Id no. & K no. & $<R V>$ & $\sigma_{<R V>}$ & $n$ & $\sigma_{\mathrm{E}}$ & $\sigma_{\mathrm{C}}$ & $O / C$ & $P\left(\chi^{2}\right)$ & $\Delta R V$ & $\Delta T$ & $v \sin i$ & $\sigma_{v \sin i}$ & Remarks \\
\hline 60 & 1752 & 1.92 & 0.24 & 4 & 0.22 & 0.48 & 0.45 & 0.898 & 0.6 & 1086 & 1.9 & & \\
\hline 61 & 1755 & 12.08 & 0.58 & 4 & 1.17 & 0.50 & 2.25 & 0.002 & 2.7 & 423 & 6.4 & 3.0 & \\
\hline $62(\mathrm{~A})$ & 1845 & 4.36 & 2.21 & 4 & 7.40 & 0.47 & 18.44 & 0.000 & 20.5 & 1122 & & & * \\
\hline $63(\mathrm{~B})$ & 1910 & -20.81 & 0.17 & 4 & 0.19 & 0.34 & 0.56 & 0.821 & 0.4 & 665 & 1.6 & & \\
\hline $64(\mathrm{C})$ & 1891 & -6.85 & 6.37 & 4 & 12.75 & 0.52 & 20.80 & 0.000 & 35.4 & 665 & 16.7 & 1.2 & $\mathrm{M}$ \\
\hline $65(\mathrm{D})$ & 1903 & 18.13 & 0.56 & 3 & 0.97 & 0.56 & 1.67 & 0.062 & 1.8 & 663 & 8.1 & 2.8 & \\
\hline $66(\mathrm{E})$ & 1984 & 32.16 & 2.83 & 4 & 5.67 & 0.38 & 14.85 & 0.000 & 11.4 & 742 & 4.5 & 1.7 & \\
\hline $67(\mathrm{~F})$ & 1991 & -0.31 & 0.32 & 4 & 0.63 & 0.57 & 1.09 & 0.317 & 1.4 & 664 & 4.1 & 2.8 & \\
\hline $68(\mathrm{G})$ & 1973 & 15.29 & 0.35 & 3 & 0.37 & 0.59 & 0.62 & 0.686 & 0.8 & 664 & 6.0 & 3.3 & \\
\hline $70(\mathrm{I})$ & 1050 & 1.41 & 0.30 & 4 & 0.59 & 0.41 & 1.45 & 0.104 & 1.3 & 700 & 1.0 & 3.1 & \\
\hline $71(\mathrm{~J})$ & 1150 & 6.71 & 0.22 & 3 & 0.31 & 0.37 & 0.83 & 0.501 & 0.6 & 739 & 1.6 & 2.7 & \\
\hline $74(\mathrm{~N})$ & 1639 & 1.41 & 0.32 & 5 & 0.45 & 0.67 & 0.63 & 0.808 & 1.1 & 1138 & 10.1 & 1.9 & \\
\hline $76(\mathrm{P})$ & 917 & 36.89 & 0.16 & 5 & 0.26 & 0.36 & 0.70 & 0.752 & 0.5 & 738 & 3.1 & 2.3 & \\
\hline $77(\mathrm{Q})$ & 1916 & -7.24 & 0.46 & 3 & 0.79 & 0.64 & 1.18 & 0.248 & 1.5 & 358 & 8.8 & 2.6 & \\
\hline 1079 & 1811 & 10.73 & 0.76 & 2 & 1.07 & 0.67 & 1.46 & 0.145 & 1.5 & 249 & 11.9 & 3.8 & \\
\hline 1083 & 1699 & 3.52 & 0.41 & 3 & 0.71 & 0.51 & 1.39 & 0.147 & 1.3 & 771 & 1.6 & 4.9 & \\
\hline 1085 & 1534 & 0.87 & 0.52 & 3 & 0.91 & 0.54 & 1.64 & 0.073 & 2.0 & 770 & 6.1 & 3.0 & \\
\hline 1092 & 1458 & 7.01 & 0.27 & 3 & 0.46 & 0.41 & 1.12 & 0.293 & 1.0 & 380 & 3.4 & & \\
\hline 2032 & 1572 & -14.32 & 0.54 & 1 & 0.52 & 0.52 & & & & & 6.9 & 4.3 & \\
\hline 2040 & 1840 & -15.84 & 0.51 & 1 & 0.51 & 0.51 & & & & & 2.2 & 6.9 & \\
\hline 2062 & 1624 & 1.98 & 0.94 & 5 & 2.09 & 0.84 & 2.09 & 0.002 & 6.3 & 1119 & 17.3 & 1.5 & \\
\hline 2076 & 2076 & 56.65 & 0.65 & 2 & 0.92 & 0.46 & 2.01 & 0.044 & 1.3 & 1118 & 2.3 & 4.8 & \\
\hline 2085 & 1632 & -1.39 & 0.25 & 3 & 0.36 & 0.42 & 0.83 & 0.510 & 0.7 & 770 & 5.2 & 2.5 & \\
\hline 2093 & 1488 & -10.51 & 1.84 & 2 & 2.60 & 0.52 & 4.95 & 0.000 & 3.7 & 247 & 2.1 & & \\
\hline 2094 & 1438 & -7.21 & 6.62 & 2 & 9.36 & 0.51 & 17.66 & 0.000 & 13.9 & 252 & 7.5 & 4.2 & \\
\hline 2095 & 1433 & 9.11 & 0.66 & 1 & 0.64 & 0.64 & & & & & 6.3 & 4.5 & \\
\hline 2096 & 1411 & -1.86 & 0.38 & 4 & 0.76 & 0.42 & 1.80 & 0.021 & 1.8 & 532 & 1.0 & 3.0 & \\
\hline 2098 & 1447 & 32.29 & 0.35 & 2 & 0.24 & 0.47 & 0.49 & 0.627 & 0.3 & 1121 & 6.6 & 2.2 & \\
\hline 2117 & 1852 & -22.12 & 0.30 & 2 & 0.43 & 0.38 & 1.12 & 0.265 & 0.6 & 775 & 3.9 & & \\
\hline 2118 & 1800 & 0.59 & 0.26 & 4 & 0.44 & 0.51 & 0.84 & 0.556 & 1.0 & 1122 & 6.2 & 1.9 & \\
\hline 2125 & 1678 & 12.06 & 0.58 & 2 & 0.82 & 0.59 & 1.37 & 0.171 & 1.2 & 1122 & 5.3 & 3.6 & \\
\hline 3001 & 1353 & 3.62 & 0.41 & 6 & 1.01 & 0.69 & 1.25 & 0.178 & 2.9 & 1481 & 16.8 & 1.2 & \\
\hline 3022 & 1265 & -40.80 & 0.57 & 1 & 0.53 & 0.53 & & & & & 10.7 & 5.6 & \\
\hline 3028 & 1031 & 52.49 & 0.60 & 1 & 0.59 & 0.59 & & & & & 4.6 & 4.7 & \\
\hline 3040 & 1026 & 23.59 & 0.65 & 1 & 0.60 & 0.60 & & & & & 12.0 & 4.0 & \\
\hline 3064 & 1097 & -22.10 & 1.06 & 1 & 0.97 & 0.97 & & & & & 12.0 & 6.5 & \\
\hline 3095 & 1008 & 1.07 & 2.99 & 2 & 4.23 & 1.40 & 2.36 & 0.019 & 6.0 & 1116 & 21.2 & 6.6 & \\
\hline 3104 & 1203 & 56.65 & 0.25 & 2 & 0.22 & 0.36 & 0.61 & 0.542 & 0.3 & 1111 & 2.4 & & \\
\hline 3105 & 1204 & 6.00 & 0.49 & 1 & 0.48 & 0.48 & & & & & 5.6 & & \\
\hline 3113 & 1362 & 124.39 & 0.51 & 1 & 0.51 & 0.51 & & & & & 4.4 & 4.9 & \\
\hline 4002 & 1373 & 0.16 & 1.47 & 6 & 3.60 & 0.97 & 2.24 & 0.000 & 10.8 & 1472 & 32.4 & 1.8 & \\
\hline 4011 & 1181 & 63.06 & 0.50 & 1 & 0.50 & 0.50 & & & & & 3.8 & 4.9 & \\
\hline 4015 & 1127 & 25.86 & 0.30 & 2 & 0.32 & 0.43 & 0.75 & 0.452 & 0.5 & 401 & 2.7 & 3.1 & \\
\hline 4027 & 925 & -15.57 & 0.40 & 3 & 0.70 & 0.50 & 1.36 & 0.158 & 1.3 & 398 & 7.6 & 2.1 & \\
\hline 4028 & 936 & 0.96 & 0.65 & 6 & 1.04 & 1.10 & 0.65 & 0.839 & 2.8 & 1156 & 27.1 & 2.2 & \\
\hline 4081 & 952 & -0.08 & 0.71 & 5 & 1.60 & 0.51 & 2.89 & 0.000 & 4.0 & 811 & 11.2 & 1.3 & \\
\hline 4088 & 1045 & 9.49 & 0.35 & 2 & 0.44 & 0.50 & 0.88 & 0.379 & 0.6 & 95 & 0.9 & 4.5 & \\
\hline 4094 & 1164 & -0.18 & 0.19 & 5 & 0.43 & 0.31 & 1.41 & 0.096 & 1.3 & 779 & 1.9 & & $\mathrm{M}^{*}$ \\
\hline 4106 & 1268 & -9.68 & 0.51 & 3 & 0.88 & 0.73 & 1.04 & 0.357 & 1.7 & 402 & 16.4 & 1.7 & \\
\hline 4114 & 1133 & 1.78 & 0.47 & 4 & 0.94 & 0.55 & 1.63 & 0.050 & 1.8 & 776 & 8.6 & 2.0 & \\
\hline 4116 & 1084 & 33.25 & 0.61 & 1 & 0.60 & 0.60 & & & & & 3.4 & 5.5 & \\
\hline 4121 & 967 & 4.56 & 1.51 & 3 & 2.61 & 0.96 & 2.12 & 0.012 & 5.2 & 379 & 21.1 & 2.4 & \\
\hline 4140 & 1357 & 17.63 & 0.84 & 2 & 1.18 & 0.58 & 2.02 & 0.044 & 1.7 & 95 & 3.6 & 3.8 & \\
\hline 6028 & & 2.29 & 0.20 & 5 & 0.94 & 0.83 & 13.92 & 0.000 & 31.4 & 668 & 21.0 & & * \\
\hline 7020 & & 48.30 & 0.40 & 1 & 0.39 & 0.39 & & & & & 3.0 & & \\
\hline
\end{tabular}

$\left(\sigma_{\mathrm{C}}\right.$ in Table 2$)$ by a factor of $\left(1+(v \sin i / 40)^{2}\right)$. In other words, we increase the raw $\sigma_{\mathrm{C}}$ by a quadratic factor which doubles the estimated value of $\sigma_{\mathrm{C}}$ by $v \sin i=40 \mathrm{~km} \mathrm{~s}^{-1}$. This procedure effectively amounts to dividing the raw $O / C$ values shown in Fig. 10 by the curve shown in the same fiure and yields the revised $O / C$ values given in Table 2. We have also applied this factor before computing the values of $\chi^{2}$ given in Table 2 and used in judging the constancy or otherwise of the radial velocity.

In computing the standard error estimates for the mean velocities given in Table 2 , we have taken into account the values of $\sigma_{\mathrm{C}}$ as well as the dispersion among the individual velocities $\left(\sigma_{\mathrm{C}}\right)$. Thus, fortuitously close agreement between a few observations $(O / C<1)$ will not lead 
to an unduly sanguine view of the accuracy of their mean (cf. also Andersen \& Nordström 1983).

For spectroscopic binaries, the mean velocity given in Table 2 has been computed from the available observations and thus appears with fairly large errors. Ideally, their full spectroscopic orbits should be determined. Obtaining the necessary 15-25 radial-velocity observations per star distributed over several years is, however, a major task and beyond the primary goal of this study, since binaries will be excluded from the isochrone fits in any case. For double-lined binaries (SB2), the systemic velocity (with errors, plus the mass ratio) can be computed with good accuracy by the method of Wilson (1941) and is then given in Table 2 instead of a simple mean.

\section{Conclusions}

With the data in this paper, NGC 3680 has been brought to a degree of completeness in both photometry, radial velocities, and proper motions previously reserved for a handful of standard clusters such as the Hyades, Pleaides, and a very few more. These data allow a fairly complete discussion of the cluster's membership, constraints on theoretical isochrones imposed by the CMD, age, and dynamical state. These topics are the subject of Paper II in this series (Nordström et al. 1996).

Acknowledgements. We are particularly indebted to our late friend Antoine Duquennoy for his competent and unselfish help with the reduction of the CORAVEL observations, always cheerfully given. We also acknowledge the collaboration of Michel Mayor and Jean-Claude Mermilliod in the study of the red giants in this and several other clusters. Barbara Anthony-Twarog and Bruce Twarog kindly provided preprints of their papers and unpublished finding charts and coordinate lists that were of much help in the CORAVEL observations, and spent much effort to help elucidate the photometric problems described in Sect. 2.2. We thank Poul Jensen for helping to extract coordinates for the stars from the GSC. Financial and other support for this work from the Carlsberg Foundation, the Danish Natural Science Research Council, the Danish Board for Astronomical Research, and the Smithsonian Institution (to BN and JA when this work was started) is gratefully acknowledged.

\section{References}

Andersen J., Andersen M.I., Klougart J., et al., 1995a, ESO Messenger 79, 12
Andersen J., Nordström B., Ardeberg A., et al., 1985, A\&AS 59,15

Andersen J., Nordström B., 1983, A\&A 122, 23

Andersen J., Nordström B., 1991, in: The Formation and Evolution of Star Clusters, Janes K. (ed. ), ASPC 13, p. 357

Andersen M.I., Storm J., Freyhammer L.M., 1995b, in: Understanding and Calibrating HST and ESO Instruments, Benvenuti P., (ed. ), p. 87

Anthony-Twarog B.J., Heim E.A., Twarog B.A., Caldwell N., 1991, AJ 102, 1056 (AHTC)

Anthony-Twarog B.J., Twarog B.A., Shodhan S., 1989, AJ 98, 1634 (ATTS)

Baranne A., Mayor M., Poncet J.-L., 1979, Vistas. Astron. 23, 279

Benz W., Mayor M., 1981, A\&A 93, 235

Benz W., Mayor M., 1984, A\&A 138, 183

Crawford D.L., Perry C.L., 1966, AJ 71, 206

Daniel S.A., Latham D.W., Mathieu R.L., Twarog B.A., 1994, PASP 106, 281

Demarque P., Sarajedini A., Guo X.-J., 1994, ApJ 426, 165

Duquennoy A., Mayor M., 1991, A\&A 248, 485

Eggen O.J., 1969, ApJ 155, 439 (EG)

Grønbech B., Olsen E.H., Strömgren B., 1976, A\&AS 26, 155

Janes K.A., Phelps R.L., 1994, AJ 108, 1773

Kozhurina-Platais V., Girard T.M., Platais I., van Altena W.F., 1995, AJ 109, 672

Lasker B.M., Sturch C.R., McLean B.J., Russel J.L., Jenkner H., Shara M.M., 1990, The Guide Star Catalog. I. Astronomical and Algorithmic Foundations, AJ 99, 2019

Mathieu R.D., Latham D.W., 1986, AJ 92, 1364

Mathieu R.D., Latham D.W., Griffin R.F., Gunn J.E., 1986, AJ 92,1100

Mayor M., 1985, in: Stellar Radial Velocities, Philip A.G.D., Latham D.W. (eds.) IAU Colloq. No. 88. L. Davis Press, Schenectady, p. 35

Mayor M., Maurice E., 1985, in: Stellar Radial Velocities, Philip A.G.D., Latham D.W. (eds.) IAU Colloq. No. 88. L. Davis Press, Schenectady, p. 299

Mermilliod J.-C., Andersen J., Nordström B., Mayor M., 1995, A\&A 299, 53

Nissen P.E., 1988, A\&A 199, 146 (N88)

Nissen P.E., Crawford D.L., Twarog B.A., 1987, AJ 93, 634

Nordström B., Andersen J., 1991, ESO Messenger 63, 34

Nordström B., Andersen J., Andersen M.I., 1996, A\&A, (Paper II) (to be submitted)

Stetson P.B., 1987, PASP 99, 191

VandenBerg D.A., 1983, ApJS 51, 29

VandenBerg D.A., 1985, ApJS 58, 711

Wilson O.C., 1941, ApJ 93, 29 